\title{
Étude des paliers lisses mésalignés lubrifiés par des fluides à couple de contrainte
}

\author{
Atika Kabouya ${ }^{1}$, Mustapha LahmaR ${ }^{2}$ et Benyebka Bou-Sä̈D ${ }^{3, a}$ \\ 1 Département de Génie Mécanique, Université de Skikda, Skikda 21000, Algérie \\ 2 Département de Génie Mécanique, Université de Guelma, BP 401, Guelma 24000, Algérie \\ 3 Laboratoire de Mécanique des Contacts \& des Structures, UMR 5259, INSA, Bâtiment J. d'Alembert, 69621 Villeurbanne, \\ France
}

Reçu le 6 avril 2007, accepté le 18 septembre 2007

Résumé - Une étude théorique du comportement statique et dynamique d'un palier lisse indéformable lubrifié par des huiles additivées en prenant en considération le défaut de mésalignement est présentée. Ces huiles dont le comportement rhéologique est non-newtonien sont modélisées comme des fluides à couple de contrainte. D'après la théorie des milieux continus de V. K. Stokes qui tient compte de la taille des particules, ces fluides se caractérisent par deux constantes physiques qui sont la viscosité dynamique $(\mu)$ et une deuxième caractéristique responsable des couples de contrainte dans le fluide $(\eta)$. Les effets des couples de contrainte sur le comportement statique et dynamique du palier aligné ou mésaligné sont étudiés en définissant un paramètre du couple de contrainte adimensionnel. Les résultats obtenus montrent que la présence des additifs dans le lubrifiant a une influence non négligeable sur les performances statiques et dynamiques ainsi que la stabilité et la réponse dynamique du palier surtout pour les grandes valeurs du paramètre du couple de contrainte, c'est-à-dire dans le cas des polymères de longue chaîne moléculaire. Toutefois, leur influence sur le débit de fuite est négligeable sauf pour les grandes excentricités de fonctionnement.

Mots clés : Paliers lisses / lubrification hydrodynamique / fluide à couple de contrainte / théorie de V. K. Stokes / caractéristiques statiques / caractéristiques dynamiques / stabilité / réponse dynamique / défaut de mésalignement

\begin{abstract}
Analysis of misaligned plain journal bearings lubricated with couple-stress fluids. A theoretical investigation of the static and dynamic behaviour of a rigid plain journal bearing lubricated by oils containing polymer additives considering misalignment defect is presented. The lubricating oils which are non-Newtonian are modelled as couple-stress fluids. According to the V. K. Stokes' micro-continuum theory which takes into account the size of particles, these fluids are characterized by two physical constants, namely the dynamic viscosity $(\mu)$ and a second characteristic responsible for the couple stress in the fluid $(\eta)$. The effects of the couple-stress on the static and dynamic behaviour of the plain journal bearing are studied by defining of a dimensionless couple-stress parameter. The obtained results showed that the presence of additives in the lubricant has non- negligible effects on the static and dynamic performance characteristics as well as the dynamic stability and response of the journal bearing especially at high values of couple-stresses parameter, i.e. for higher chain length of the additive molecule. However, their influence on the side leakage flow is negligible except for high values of eccentricity ratio.
\end{abstract}

Key words: Plain journal bearings / hydrodynamic lubrication / couple-stress fluid / V. K. Stokes' theory / steady-state performance characteristics / dynamic characteristics / stability / dynamic response / misalignment defect

\section{Introduction}

a Auteur pour correspondance :

Benyebka.Bou-Said@insa-lyon.fr
Dans les études de simulation des problèmes de lubrification, on suppose généralement que le comportement des 


\section{Nomenclature}

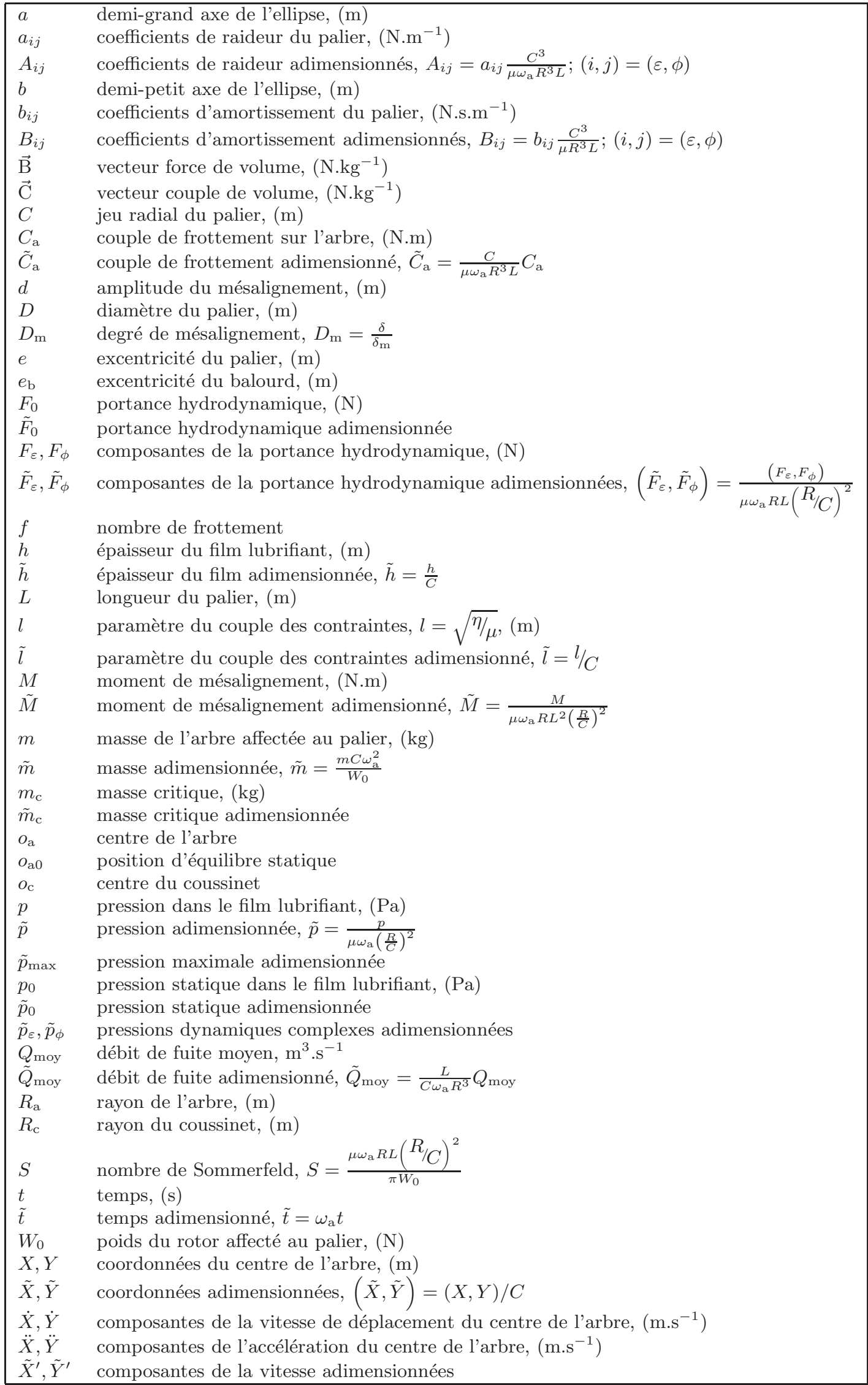




\begin{tabular}{|ll|}
\hline$\tilde{X}^{\prime \prime}, \tilde{Y}^{\prime \prime}$ & composantes de l'accélération adimensionnées \\
$z$ & coordonnée axiale du palier mesurée à partir de la section médiane, $(\mathrm{m})$ \\
$\tilde{z}$ & coordonnée axiale adimensionnée \\
$\beta$ & angle de mésalignement, (rad) \\
$\delta$ & mésalignement relatif, $\delta=d / C$ \\
$\delta^{*}$ & angle d'inclinaison du demi-grand axe de l'ellipse par rapport à l'axe $X,(\mathrm{rad})$ \\
$\varepsilon$ & excentricité relative, $\varepsilon=\frac{e}{C}$ \\
$\varepsilon_{0}$ & excentricité relative statique dans la section médiane du palier \\
$\varepsilon_{\mathrm{b}}$ & excentricité relative du balourd, $\varepsilon_{\mathrm{b}}=\frac{e_{\mathrm{b}}}{C}$ \\
$\eta$ & constante physique due à la présence des couples de contraintes, (N.s) \\
$\phi$ & angle de calage, $(\mathrm{rad})$ \\
$\varphi$ & angle de déphasage entre le vecteur balourd et le vecteur déplacement,,$(\mathrm{rad})$ \\
$\theta$ & position angulaire d'un point de la surface du coussinet, (rad) \\
$\theta_{\mathrm{c} 0}$ & angle de cavitation en régime statique, $(\mathrm{rad})$ \\
$\omega_{\mathrm{a}}$ & vitesse angulaire de l'arbre, $\left(\mathrm{rad} . \mathrm{s}^{-1}\right)$ \\
$v$ & pulsation d'excitation du palier, $\left(\mathrm{rad} . \mathrm{s}^{-1}\right)$ \\
$\rho$ & masse volumique du lubrifiant, $\left(\mathrm{kg} \cdot \mathrm{m}^{-3}\right)$ \\
$\gamma$ & pulsation d'excitation adimensionnée, $\gamma=\frac{v}{\omega_{\mathrm{a}}}$ \\
$\gamma_{\mathrm{c}}$ & pulsation de fouettement adimensionnée \\
$\mu$ & viscosité dynamique du lubrifiant, $(\mathrm{Pa} . \mathrm{s})$ \\
$\circ$ & différentiation par rapport à $t$ \\
$(\bullet)$ & différentiation par rapport à $\tilde{t},(\bullet)^{\prime}=\frac{1}{\omega}(\bullet)$ \\
$(\bullet)^{\prime}$ & quantité adimensionnée \\
$(\tilde{\bullet})$ & quantité vectorielle \\
$(\vec{\bullet})$ &
\end{tabular}

fluides lubrifiants est newtonien. Néanmoins, les applications pratiques de la lubrification montrent que le modèle de fluide newtonien ne constitue pas une approche satisfaisante des problèmes de lubrification avec des huiles dopées ou additivées. Des études expérimentales ont montré que les huiles contenant des additifs de viscosité ont un comportement rhéologique non-newtonien, leur viscosité diminue quand le taux de cisaillement auquel elles sont soumises augmente. Ainsi, leur écoulement ne peut être décrit par la théorie des milieux continus classique qui néglige la taille des particules. Dans la littérature, il existe plusieurs théories permettant de décrire l'écoulement de ces fluides dits à rhéologie complexe [1-3]. Parmi ces théories, la théorie des milieux continus de Vijay Kumar Stokes [1] est la plus simple et la plus utilisée car elle permet de tenir compte de la taille des particules en mouvement et des couples de volume et de contrainte dus à la présence des additifs. Dans la présente étude, le lubrifiant est assimilé à un fluide incompressible à couple de contrainte pour lequel seuls les moments de contrainte qui s'ajoutent aux forces de surface (forces de pression et de frottement visqueux) sont pris en considération. Le modèle de fluide à couple de contrainte a été largement utilisé pour l'étude de certains problèmes de la lubrification hydrodynamique et hydrostatique. Lin [4-6] a étudié les effets du paramètre du couple de contrainte $\tilde{l}=\frac{l}{C}$ sur les caractéristiques du film amortisseur d'un palier à arc partiel et d'un palier complet de longueur finie en appliquant la théorie des milieux continus de V. K. Stokes. Les résultats théoriques obtenus montrent que la présence des couples de contrainte permet d'avoir une amélioration de la capacité de charge du palier. Oliver [7] a montré expérimentalement que la présence de polymères dissous dans les lubrifiants entraîne une augmentation de la capacité de charge du film lubrifiant et une diminution du coefficient de frottement. Dans une autre étude, Mokhiamar et al. [8] ont étudié l'effet du paramètre du couple de contrainte sur les caractéristiques statiques d'un palier lisse en considérant l'élasticité du revêtement de surface. Ils ont montré qu'une augmentation du paramètre du couple de contrainte entraîne :

- une augmentation du pic de pression et de la portance du palier, celle-ci est plus importante à forte excentricité;

- une diminution de l'angle de calage et du coefficient de frottement.

Ils montrent par ailleurs que le débit de fuite n'est pas influencé, sauf pour les grandes valeurs du coefficient d'élasticité; c'est-à-dire dans le cas de revêtements caractérisés par de faibles valeurs du module d'élasticité. Les mêmes constatations ont également été faites plus récemment par Lahmar [9] dans le cas d'un palier bicouche.

Lin [10] a étudié l'influence du paramètre du couple de contrainte sur la stabilité linéaire d'un palier lisse rigide. Il a montré que le système lubrifié par un fluide à couple de contrainte est plus stable que celui lubrifié par un fluide newtonien.

Il convient de noter que toutes les études précédentes ont été effectuées en régime de lubrification isotherme : la température est supposée constante dans les deux milieux fluide et solide. Wang et al. [11] ont utilisé une approche thermohydrodynamique pour étudier le comportement d'un palier lisse soumis à une charge statique lubrifié par un fluide à couple de contrainte. Ils ont conclu que la 
température du palier calculée dans le cas du fluide à couple de contrainte est plus faible que celle obtenue pour un fluide newtonien (non polaire). Dans un autre travail, les mêmes auteurs ont utilisé le même modèle de fluide pour étudier le comportement dynamique du palier de bielle d'un moteur à combustion interne [12].

L'application de la théorie de V. K. Stokes [1] et des lois fondamentales de la mécanique des milieux continus à l'étude des écoulements de films minces a permis de dériver une équation aux dérivées partielles linéaire de type elliptique appelée équation de Reynolds modifiée dans le cas d'un palier fonctionnant en régime dynamique. Dans le cas où le rotor est soumis à des vibrations harmoniques de faible amplitude au voisinage de la position d'équilibre statique, la méthode de perturbation d'ordre un permet de transformer l'équation de Reynolds modifiée en un système de trois équations différentielles partielles linéaires couplées dont les inconnues principales sont les pressions statiques et dynamiques. Le système d'équations d'ordres zéro et un ainsi obtenu est résolu par la méthode des différences finies en vue de déterminer les caractéristiques statiques et dynamiques du palier, à savoir la portance hydrodynamique, l'angle de calage, le nombre de frottement, le débit de fuite et les coefficients de raideur et d'amortissement. Ces coefficients servent comme des données pour le calcul de la frontière de stabilité et l'étude de la réponse du palier à un balourd résiduel.

Dans ce travail basé sur l'application de la théorie des milieux continus de V. K. Stokes, on s'intéresse à l'étude théorique de l'influence du paramètre du couple de contrainte sur les performances statiques et dynamiques des paliers lisses indéformables en prenant en considération le mésalignement de l'arbre dans le coussinet. Le paramètre du couple de contrainte dont la valeur s'échelonne entre 0 et 1 représente physiquement la longueur maximale relative des chaînes moléculaires des additifs rajoutés à l'huile de base en vue d'améliorer les performances du lubrifiant.

\section{2 Équations géométriques du film lubrifiant}

\subsection{Cas du palier aligné}

La figure 1 représente schématiquement la section droite d'un palier cylindrique. Dans le cas d'un palier aligné, l'épaisseur du film d'huile est calculée par la relation suivante :

$$
h=C(1+\varepsilon \cos \theta)
$$

où $C$ est le jeu radial du palier, $\theta$ la coordonnée circonférentielle mesurée à partir de la ligne des centres et $\varepsilon=\frac{e}{C}$ l'excentricité relative variant de 0 à 1 .

\subsection{Cas du palier mésaligné}

Dans le cas du palier mésaligné (Fig. 2), l'excentricité $e$ ainsi que l'angle de calage $\phi$ sont fonctions de la

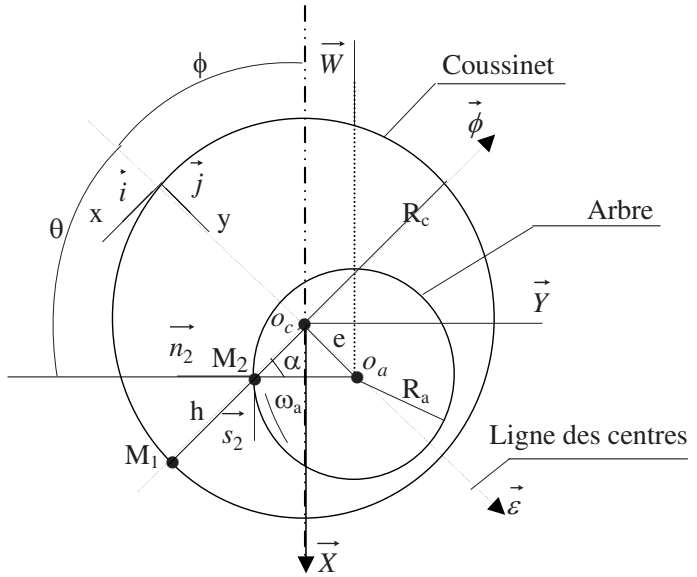

Fig. 1. Systèmes d'axes.
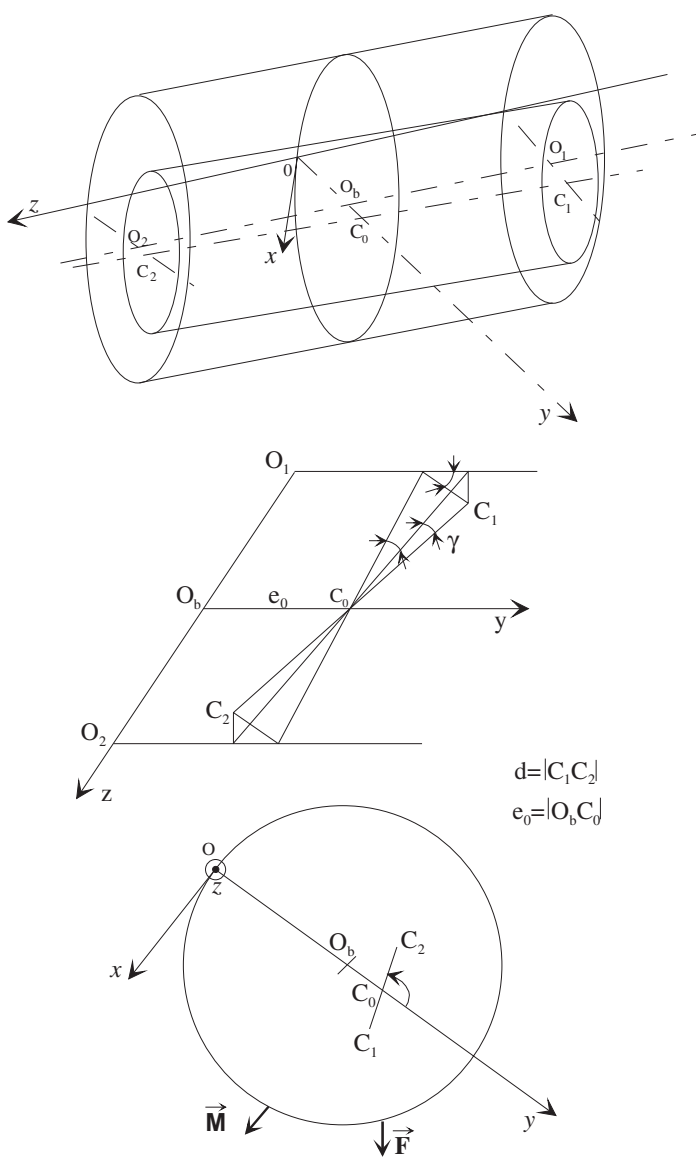

Fig. 2. Palier mésaligné.

coordonnée axiale $z$. Dans ces conditions, l'épaisseur du film d'huile est calculée par [13] :

$$
h(\theta, z)=C\left(1+\varepsilon \cos \theta+\delta \frac{z}{L} \cos (\theta-\beta)\right)
$$

où $\delta=\frac{d}{C}$ est le mésalignement relatif, $\beta$ l'angle de mésalignement, $L$ la longueur du palier. Dans l'expression de $\delta, d$ est l'amplitude de mésalignement caractérisée par le module de la projection de l'axe $C_{1} C_{2}$ de l'arbre 
sur la section médiane. Le domaine de variation de ces paramètres est :

$$
-180^{\circ} \leqslant \beta \leqslant 180^{\circ} ; 0<\varepsilon<1 ; 0 \leqslant \delta \leqslant \delta_{\mathrm{m}}
$$

$\delta_{\mathrm{m}}$ représente la valeur maximale que peut prendre $\delta$ pour une excentricité et un angle de mésalignement fixés dont l'expression est :

$$
\delta_{\mathrm{m}}=2\left(\sqrt{1-\varepsilon^{2} \sin ^{2} \beta}-\varepsilon|\cos \beta|\right)
$$

La valeur de $\delta_{\mathrm{m}}$ est toujours comprise entre 0 et 2 .

La symétrie du palier par rapport au plan défini par la section médiane permet de réduire l'intervalle de variation de $\beta$ à $\pm 90^{\circ}$.

\section{3 Équations de la lubrification hydrodynamique}

\section{1 Équations de mouvement du fluide polaire}

En se basant sur la théorie des milieux continus de Stokes, les équations de mouvement d'un fluide incompressible à couple de contraintes sont :

$$
\begin{gathered}
\rho \frac{D \vec{V}}{D t}=-\vec{\nabla} p+\rho \vec{B}+\frac{1}{2} \vec{\nabla} \times(\rho \vec{C})+\mu \vec{\nabla}^{2} \vec{V}-\eta \vec{\nabla}^{4} \vec{V} \\
\vec{\nabla} \cdot \vec{V}=0
\end{gathered}
$$

En négligeant les forces et les couples de volume, les équations (4) et (5) s'écrivent en coordonnées cartésiennes comme suit :

$$
\begin{aligned}
& \rho\left(u \frac{\partial u}{\partial x}+v \frac{\partial u}{\partial y}+w \frac{\partial u}{\partial z}\right)=-\frac{\partial p}{\partial x}+\mu\left(\frac{\partial^{2} u}{\partial x^{2}}+\frac{\partial^{2} u}{\partial y^{2}}+\frac{\partial^{2} u}{\partial z^{2}}\right) \\
& -\eta\left(\frac{\partial^{4} u}{\partial x^{4}}+\frac{\partial^{4} u}{\partial y^{4}}+\frac{\partial^{4} u}{\partial z^{4}}\right) \\
& -2 \eta\left(\frac{\partial^{4} u}{\partial x^{2} \partial y^{2}}+\frac{\partial^{4} u}{\partial x^{2} \partial y^{2}}+\frac{\partial^{4} u}{\partial y^{2} \partial z^{2}}\right) \\
& \rho\left(u \frac{\partial v}{\partial x}+v \frac{\partial v}{\partial y}+w \frac{\partial v}{\partial z}\right)=-\frac{\partial p}{\partial y}+\mu\left(\frac{\partial^{2} v}{\partial x^{2}}+\frac{\partial^{2} v}{\partial y^{2}}+\frac{\partial^{2} v}{\partial z^{2}}\right) \\
& -\eta\left(\frac{\partial^{4} v}{\partial x^{4}}+\frac{\partial^{4} v}{\partial y^{4}}+\frac{\partial^{4} v}{\partial z^{4}}\right) \\
& -2 \eta\left(\frac{\partial^{4} v}{\partial x^{2} \partial y^{2}}+\frac{\partial^{4} v}{\partial x^{2} \partial z^{2}}+\frac{\partial^{4} v}{\partial y^{2} \partial z^{2}}\right) \\
& \rho\left(u \frac{\partial w}{\partial x}+v \frac{\partial w}{\partial y}+w \frac{\partial w}{\partial z}\right)=-\frac{\partial p}{\partial z}+\mu\left(\frac{\partial^{2} w}{\partial x^{2}}+\frac{\partial^{2} w}{\partial y^{2}}+\frac{\partial^{2} w}{\partial z^{2}}\right) \\
& -\eta\left(\frac{\partial^{4} w}{\partial x^{4}}+\frac{\partial^{4} w}{\partial y^{4}}+\frac{\partial^{4} w}{\partial z^{4}}\right)- \\
& 2 \eta\left(\frac{\partial^{4} w}{\partial x^{2} \partial y^{2}}+\frac{\partial^{4} w}{\partial x^{2} \partial z^{2}}+\frac{\partial^{4} w}{\partial y^{2} \partial z^{2}}\right)
\end{aligned}
$$

$$
\frac{\partial u}{\partial x}+\frac{\partial v}{\partial y}+\frac{\partial w}{\partial z}=0
$$

En utilisant les hypothèses de la lubrification hydrodynamique établies pour ce type d'écoulement, les équations de mouvement se réduisent à [9] :

$$
\begin{gathered}
\frac{\partial p}{\partial x}=\mu \frac{\partial^{2} u}{\partial y^{2}}-\eta \frac{\partial^{4} u}{\partial y^{4}} \\
\frac{\partial p}{\partial y}=0 \\
\frac{\partial p}{\partial z}=\mu \frac{\partial^{2} w}{\partial y^{2}}-\eta \frac{\partial^{4} w}{\partial y^{4}}
\end{gathered}
$$

\section{2 Équation de Reynolds modifiée}

a) Conditions aux limites :

$$
\begin{aligned}
& \left\{\begin{array}{l}
u(x, o, z)=U_{1}=0 \\
w(x, o, z)=W_{1}=0
\end{array}\right. \\
& \left.\frac{\partial^{2} u}{\partial y^{2}}\right|_{y=0}=\left.\frac{\partial^{2} w}{\partial y^{2}}\right|_{y=0}=0 \\
& u(x, h, z)=U_{2} \\
& w(x, h, z)=W_{2}=0 \\
& \left.\frac{\partial^{2} u}{\partial y^{2}}\right|_{y=h}=\left.\frac{\partial^{2} w}{\partial y^{2}}\right|_{y=h}=0 \\
& \left\{\begin{array}{l}
v(x, o, z)=V_{1}=0 \\
v(x, h, z)=V_{2}
\end{array}\right.
\end{aligned}
$$

$U_{2}$ et $V_{2}$ sont les composantes normales et tangentielles de la vitesse. L'équation (10a) représente la condition limite de non glissement et les équations (9b) et (10c) représentent les conditions permettant l'annulation des couples de contraintes aux parois. L'intégration des équations de mouvement (8a) et (8c) avec l'utilisation des conditions limites correspondantes permet d'exprimer les vitesses $u$ et $w$ par :

$$
\left\{\begin{array}{l}
u=U_{2} \frac{y}{h}+\frac{1}{2 \mu} \frac{\partial p}{\partial x}\left(y(y-h)+2 l^{2}\left(1-\frac{\operatorname{ch}\left(\frac{2 y-h}{2 l}\right)}{c h\left(\frac{h}{2 l}\right)}\right)\right) \\
w=\frac{1}{2 \mu} \frac{\partial p}{\partial z}\left(y(y-h)+2 l^{2}\left(1-\frac{\operatorname{ch}\left(\frac{2 y-h}{2 l}\right)}{\operatorname{ch}\left(\frac{h}{2 l}\right)}\right)\right)
\end{array}\right.
$$

En substituant les équations de la vitesse (12) dans l'équation de continuité (7) et en utilisant les conditions aux limites (11), cela permet de dériver l'équation de Reynolds modifiée :

$$
\begin{aligned}
& \frac{\partial}{\partial x}\left(G(h, l) \frac{\partial p}{\partial x}\right)+ \frac{\partial}{\partial z}\left(G(h, l) \frac{\partial p}{\partial z}\right)= \\
& 6 \mu\left(\frac{\partial}{\partial x}\left(h U_{2}\right)-2 U_{2} \frac{\partial h}{\partial x}+2 V_{2}\right)
\end{aligned}
$$


où

$$
G(h, l)=h^{3}-12 l^{2} h+24 l^{3} t h\left(\frac{h}{2 l}\right)
$$

Dans l'annexe A, on montre que les composantes du vecteur vitesse de la surface de l'arbre s'expriment par :

$$
\left\{\begin{aligned}
U_{2}= & \omega_{\mathrm{a}} R+C \dot{\varepsilon} \sin \theta-C \varepsilon \dot{\phi} \cos \theta \\
& -\frac{\partial h}{\partial x}(C \dot{\varepsilon} \cos \theta+C \varepsilon \dot{\phi} \sin \theta) \\
V_{2}= & \omega_{\mathrm{a}} R \frac{\partial h}{\partial x}+C \dot{\varepsilon} \cos \theta+C \varepsilon \dot{\phi} \sin \theta \\
& +\frac{\partial h}{\partial x}(C \dot{\varepsilon} \sin \theta-C \varepsilon \dot{\phi} \cos \theta)
\end{aligned}\right.
$$

En régime permanent, les composantes du vecteur vitesse (15) se réduisent à :

$$
\left\{\begin{array}{l}
U_{2}=\omega_{\mathrm{a}} R \\
V_{2}=U_{2} \frac{\partial h}{\partial x}
\end{array}\right.
$$

\section{3 Équation de Reynolds modifiée en régime dynamique}

Puisque $\left(\frac{\partial h}{\partial x}\right)^{2}<<1$, le second membre de l'équation de Reynolds modifiée (13) se simplifie comme suit :

$$
6 \mu\left(-U_{2} \frac{\partial h}{\partial x}+2 V_{2}\right) \approx 6 \mu\left(\frac{\partial h}{\partial \theta}\left(\omega_{\mathrm{a}}-2 \dot{\phi}\right)+2 C \dot{\varepsilon} \cos \theta\right)
$$

Par conséquent, l'équation de Reynolds modifiée écrite pour un palier lisse est :

$$
\begin{aligned}
& \frac{\partial}{\partial x}\left(G(h, l) \frac{\partial p}{\partial x}\right)+\frac{\partial}{\partial z}\left(G(h, l) \frac{\partial p}{\partial z}\right)= \\
& 6 \mu\left(\frac{\partial h}{\partial \theta}\left(\omega_{\mathrm{a}}-2 \dot{\phi}\right)+2 C \dot{\varepsilon} \cos \theta\right)
\end{aligned}
$$

Pour le traitement numérique, on doit écrire l'équation de Reynolds en variables sans dimension. Pour cela, on pose : $\theta=\frac{x}{R} ; \tilde{z}=\frac{z}{L} ; \tilde{h}=\frac{h}{C} ; \tilde{t}=\omega_{\mathrm{a}} t ; \tilde{p}=\frac{p}{\mu \omega_{\mathrm{a}}\left(\frac{R}{C}\right)^{2}}$; $\tilde{l}=\frac{l}{C} ; \tilde{G}(\tilde{h}, \tilde{l})=\frac{G(h, l)}{C^{3}}$.

Il vient alors :

$$
\begin{aligned}
\frac{\partial}{\partial \theta}\left(\tilde{G}(\tilde{h}, \tilde{l}) \frac{\partial \tilde{p}}{\partial \theta}\right) & +\left(\frac{R}{L}\right)^{2} \frac{\partial}{\partial \tilde{z}}\left(\tilde{G}(\tilde{h}, \tilde{l}) \frac{\partial \tilde{p}}{\partial \tilde{z}}\right)= \\
& 6\left(\frac{\partial \tilde{h}}{\partial \theta}\left(1-2 \frac{\mathrm{d} \phi}{\mathrm{d} \tilde{t}}\right)+2 \frac{\mathrm{d} \varepsilon}{\mathrm{d} \tilde{t}} \cos \theta\right)
\end{aligned}
$$

\subsection{Application de la méthode de perturbation}

Lorsque l'arbre est soumis à des vibrations harmoniques de faible amplitude au voisinage de la position d'équilibre statique $O_{\mathrm{a} 0}$ définie par les coordonnées $\left(\varepsilon_{0}, \phi_{0}\right)$, l'excentration et l'angle de calage instantanés peuvent être exprimés par [9] :

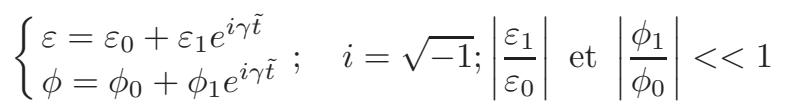

où $\gamma=\frac{v}{\omega_{\mathrm{a}}}$ est la fréquence d'excitation adimensionnée, et $\varepsilon_{1}$ et $\phi_{1}$, sont respectivement les amplitudes de perturbation de l'excentration et de l'angle de calage. Dans ces conditions, l'épaisseur du film lubrifiant et le champ de pression peuvent être exprimés par :

$$
\left\{\begin{array}{l}
\tilde{h}=\tilde{h}_{0}+\tilde{h}_{1} e^{i \gamma \tilde{t}} \\
\tilde{p}=\tilde{p}_{0}+\underbrace{\left(\varepsilon_{1} \tilde{p}_{\varepsilon}+\varepsilon_{0} \phi_{1} \tilde{p}_{\phi}\right)}_{\tilde{p}_{1}} e^{i \gamma \tilde{t}}
\end{array}\right.
$$

où $\tilde{h}_{0}=1+\varepsilon_{0} \cos \theta$ est l'épaisseur du film adimensionnée à la position d'équilibre statique, $\tilde{h}_{1}=\varepsilon_{1} \cos \theta+\varepsilon_{0} \phi_{1} \sin \theta$ est l'amplitude de déplacement du centre de l'arbre dû à la vibration, $\tilde{p}_{0}$ représente le champ de pression sans dimension en régime stationnaire, et $\tilde{p}_{\varepsilon}$ et $\tilde{p}_{\phi}$ sont des pressions dynamiques complexes telles que $\tilde{p}_{\varepsilon}=\frac{\partial \tilde{p}}{\partial \varepsilon}$ et $\tilde{p}_{\phi}=\frac{1}{\varepsilon_{0}} \frac{\partial \tilde{p}}{\partial \phi}$.

En remplaçant les équations (21) dans l'équation (19) et en utilisant l'opérateur $\Re(\bullet)=$ $\frac{\partial}{\partial \theta}\left(\tilde{G}_{0}\left(\tilde{h}_{0}, \tilde{l}\right) \frac{\partial}{\partial \theta}(\bullet)\right)+\left(\frac{R}{L}\right)^{2} \frac{\partial}{\partial \tilde{z}}\left(\tilde{G}_{0}\left(\tilde{h}_{0}, \tilde{l}\right) \frac{\partial}{\partial \tilde{z}}(\bullet)\right)$, on trouve :

- équation d'ordre zéro

$$
\Re\left(\tilde{p}_{0}\right)=6 \frac{\partial \tilde{h}_{0}}{\partial \theta}
$$

- équation d'ordre un

$$
\begin{aligned}
\Re\left(\tilde{p}_{\varepsilon}\right) & +18 \frac{\tilde{h}_{0}^{2} \cos \theta}{\tilde{G}_{0}} \frac{\partial \tilde{h}_{0}}{\partial \theta}+3 \tilde{G}_{0} \frac{\partial \tilde{p}_{0}}{\partial \theta} \frac{\partial f}{\partial \theta} \\
& +3\left(\frac{R}{L}\right)^{2} \tilde{G}_{0} \frac{\partial \tilde{p}_{0}}{\partial \tilde{z}} \frac{\partial f}{\partial \tilde{z}}=-6 \sin \theta+12 i \gamma \cos \theta
\end{aligned}
$$

$$
\begin{aligned}
\Re\left(\tilde{p}_{\phi}\right)+ & 18 \frac{\tilde{h}_{0}^{2} \sin \theta}{\tilde{G}_{0}} \frac{\partial \tilde{h}_{0}}{\partial \theta}+3 \tilde{G}_{0} \frac{\partial \tilde{p}_{0}}{\partial \theta} \frac{\partial g}{\partial \theta} \\
& +3\left(\frac{R}{L}\right)^{2} \tilde{G}_{0} \frac{\partial \tilde{p}_{0}}{\partial \tilde{z}} \frac{\partial g}{\partial \tilde{z}}=6 \cos \theta+12 i \gamma \sin \theta
\end{aligned}
$$

avec $\tilde{G}_{0}\left(\tilde{h}_{0}, \tilde{l}\right)=\tilde{h}_{0}^{3}-12 \tilde{l}^{2} \tilde{h}_{0}+24 \tilde{l}^{3} t h\left(\frac{\tilde{h}_{0}}{2 \tilde{l}}\right) ; f(\theta, \tilde{z})=$ $\frac{\tilde{h}_{0}^{2} \cos \theta}{\tilde{G}_{0}}$ et $g(\theta, \tilde{z})=\frac{\tilde{h}_{0}^{2} \sin \theta}{\tilde{G}_{0}}$

Les conditions limites sur la pression sont :

- Conditions liées à l'environnement du palier :

$$
\begin{gathered}
\tilde{p}_{0}\left(\theta, \tilde{z}= \pm \frac{1}{2}\right)=0 \\
\tilde{p}_{\varepsilon}\left(\theta, \tilde{z}= \pm \frac{1}{2}\right)=\tilde{p}_{\phi}\left(\theta, \tilde{z}= \pm \frac{1}{2}\right)=0
\end{gathered}
$$

- Conditions liées à l'écoulement du fluide lubrifiant :

$$
\tilde{p}_{0}=\frac{\partial \tilde{p}_{0}}{\partial \theta}=0 \text { pour } \theta=\theta_{c 0}
$$


La condition (23c) permet de prendre en considération la rupture du film dans la région divergente du palier. Dans les calculs, la prise en considération de cette condition est assurée à l'aide de l'algorithme de Christopherson [14]. L'application de cet algorithme permet d'annuler au cours des calculs les termes de pression négatifs. Il est à noter que le domaine d'intégration des équations d'ordre un est celui défini par l'application de l'algorithme de Christopherson lors de la résolution de l'équation de Reynolds statique d'ordre zéro. D'autre part, les équations dynamiques (22b) et (22c) doivent être résolues sans annulation des pressions négatives.

\section{5 Étude statique du palier}

La détermination de la répartition de la pression statique dans le film lubrifiant $\tilde{p}_{0}$ pour une position de l'arbre dans le palier permet de déterminer les performances statiques du palier.

\section{a) Portance hydrodynamique}

Dans le repère intermédiaire $\left(o_{\mathrm{c}}, \vec{\varepsilon}, \vec{\phi}, \vec{z}\right)$, les composantes de la portance en variables sans dimension s'expriment par :

$$
\left\{\begin{array}{l}
\tilde{F}_{\varepsilon}=\int_{-\frac{1}{2}}^{\frac{1}{2}} \int_{0}^{2 \pi} \tilde{p}_{0}(\theta, \tilde{z}) \cos \theta \mathrm{d} \theta \mathrm{d} \tilde{z} \\
\tilde{F}_{\phi}=\int_{-\frac{1}{2}}^{\frac{1}{2}} \int_{0}^{2 \pi} \tilde{p}_{0}(\theta, \tilde{z}) \sin \theta \mathrm{d} \theta \mathrm{d} \tilde{z}
\end{array}\right.
$$

De ces deux relations, on peut déterminer :

- l'angle de calage statique $\phi_{0}$

$$
\phi_{0}=\tan ^{-1}\left(-\frac{\tilde{F}_{\phi}}{\tilde{F}_{\varepsilon}}\right)
$$

- la résultante hydrodynamique $\tilde{F}_{0}$

$$
\tilde{F}_{0}=\sqrt{\tilde{F}_{\varepsilon}^{2}+\tilde{F}_{\phi}^{2}}
$$

- le nombre de Sommerfeld $S$

$$
S=\frac{1}{\pi \tilde{F}_{0}}
$$

b) Couple de frottement

Le couple de frottement est obtenu par intégration des contraintes de cisaillement à la surface de l'arbre :

$$
\tilde{C}_{\mathrm{a}}=\int_{-\frac{1}{2}}^{\frac{1}{2}} \int_{0}^{2 \pi}\left(\frac{1}{\tilde{h}_{0}}+\frac{1}{2} \frac{\partial \tilde{p}_{0}}{\partial \theta}\left(\tilde{h}_{0}-2 \tilde{l} t h\left(\frac{\tilde{h}_{0}}{2 \tilde{l}}\right)\right)\right) \mathrm{d} \theta \mathrm{d} \tilde{z}
$$

c) Nombre de frottement

Le nombre de frottement est défini par :

$$
f=\frac{\tilde{C}_{\mathrm{a}}}{\tilde{F}_{0}}
$$

d) Le débit de fuite

$$
\begin{aligned}
\tilde{Q}_{\text {moy }}= & -\frac{1}{12} \int_{0}^{2 \pi} \tilde{h}_{0}^{3} \frac{\partial \tilde{p}_{0}}{\partial \tilde{z}} \mathrm{~d} \theta \\
& +\tilde{l}^{2} \int_{0}^{2 \pi} \frac{\partial \tilde{p}_{0}}{\partial \tilde{z}}\left[\tilde{h}_{0}-2 \tilde{l} t h\left(\frac{\tilde{h}_{0}}{2 \tilde{l}}\right)\right] \mathrm{d} \theta
\end{aligned}
$$

e) Moment de mésalignement

En variables adimensionnelles, les composantes du vecteur moment des forces de pression s'écrit :

$$
\begin{aligned}
\tilde{M} \sin \psi & =\int_{0}^{2 \pi} \int_{-\frac{1}{2}}^{\frac{1}{2}} \tilde{z} \tilde{p}_{0}(\theta, \tilde{z}) \cos \theta \mathrm{d} \tilde{z} \mathrm{~d} \theta \\
\tilde{M} \cos \psi & =\int_{0}^{2 \pi} \int_{-\frac{1}{2}}^{\frac{1}{2}} \tilde{z} \tilde{p}_{0}(\theta, \tilde{z}) \sin \theta \mathrm{d} \tilde{z} \mathrm{~d} \theta
\end{aligned}
$$

$\psi$ étant l'angle que fait le vecteur moment de mésalignement $\vec{M}$ avec la ligne des centres oy.

\section{6 Étude dynamique linéaire du palier}

a) Coefficients de raideur et d'amortissement

La détermination de la distribution des pressions dynamiques complexes $\tilde{p}_{\varepsilon}$ et $\tilde{p}_{\phi}$ permet de déterminer les coefficients de raideur et d'amortissement sans dimension du film :

$$
\left[\begin{array}{ll}
Z_{\varepsilon \varepsilon} & Z_{\varepsilon \phi} \\
Z_{\phi \varepsilon} & Z_{\phi \phi}
\end{array}\right]^{T}=-\int_{-\frac{1}{2}}^{\frac{1}{2}} \int_{0}^{2 \pi}\left\{\begin{array}{l}
\tilde{p}_{\varepsilon} \\
\tilde{p}_{\phi}
\end{array}\right\}<\cos \theta \vdots \sin \theta>\mathrm{d} \theta \mathrm{d} \tilde{z}
$$

où $Z_{\varepsilon \phi}=A_{\varepsilon \phi}+i \gamma B_{\varepsilon \phi}$; etc. sont les impédances complexes du palier.

Le changement de système de coordonnées permet d'exprimer les coefficients de raideur dans le repère fixe $\left(O_{\mathrm{c}}, X, Y\right)$ :

$$
\begin{aligned}
{\left[\begin{array}{ll}
A_{X X} & A_{X Y} \\
A_{Y X} & A_{Y Y}
\end{array}\right]=} & {\left[\begin{array}{ll}
\cos \phi_{0} & -\sin \phi_{0} \\
\sin \phi_{0} & \cos \phi_{0}
\end{array}\right]\left[\begin{array}{ll}
A_{r r} & A_{r t} \\
A_{t r} & A_{t t}
\end{array}\right] } \\
& \times\left[\begin{array}{ll}
\cos \phi_{0} & \sin \phi_{0} \\
-\sin \phi_{0} & \cos \phi_{0}
\end{array}\right]
\end{aligned}
$$

et des relations similaires pour les coefficients d'amortissement.

b) Système en vibrations libres : paramètres de stabilité (pulsation de fouettement et vitesse critique)

L'étude de stabilité est effectuée dans le cas d'un rotor rigide de masse $2 m$ supporté horizontalement par deux paliers lisses identiques. Dans le système de coordonnées $\left(O_{\mathrm{c}}, X, Y\right)$, les équations linéarisées du mouvement libre du rotor en variables sans dimension s'écrivent :

$$
\begin{aligned}
{\left[\begin{array}{cc}
\tilde{m} & 0 \\
0 & \tilde{m}
\end{array}\right]\left\{\begin{array}{l}
\tilde{X}^{\prime \prime} \\
\tilde{Y}^{\prime \prime}
\end{array}\right\}+} & \left\{\begin{array}{ll}
B_{X X} & B_{X Y} \\
B_{Y X} & B_{Y Y}
\end{array}\right\}\left\{\begin{array}{l}
\tilde{X}^{\prime} \\
\tilde{Y}^{\prime}
\end{array}\right\} \\
& +\left[\begin{array}{ll}
K_{X X} & K_{X Y} \\
K_{Y X} & K_{Y Y}
\end{array}\right]\left\{\begin{array}{l}
\tilde{X} \\
\tilde{Y}
\end{array}\right\}=\left\{\begin{array}{l}
0 \\
0
\end{array}\right\}
\end{aligned}
$$

La solution de ce système d'équations est recherchée sous la forme suivante :

$$
\left\{\begin{array}{l}
\tilde{X} \\
\tilde{Y}
\end{array}\right\}=\left\{\begin{array}{l}
\tilde{X}_{\mathrm{a}} \\
\tilde{Y}_{\mathrm{a}}
\end{array}\right\} e^{\lambda \tilde{t}}
$$

où $\tilde{X}_{\mathrm{a}}$ et $\tilde{Y}_{\mathrm{a}}$ sont les amplitudes du mouvement, $\lambda=\xi+i \gamma$ est une valeur propre complexe, $\xi$ représente l'amortissement structural, au seuil de la stabilité $(\xi=0)$. 


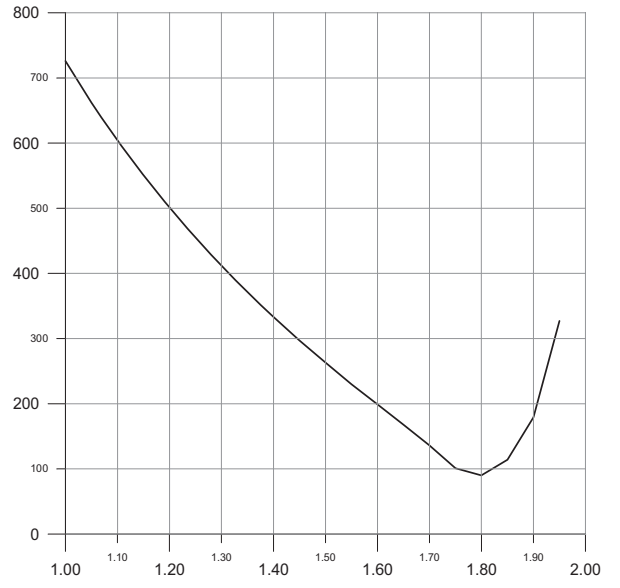

Fig. 3. Influence du coefficient de sur-relaxation sur le nombre d'itérations pour un maillage $N_{\theta}=60$ et $N_{z}=20$.

La résolution de ce système d'équations permet de calculer les paramètres de stabilité sans dimension $\tilde{m}_{\mathrm{c}}$ et $\gamma_{\mathrm{c}}$.

La vitesse angulaire critique sans dimension du rotor est calculée par :

$$
\tilde{\omega}_{\mathrm{c}}=\omega_{\mathrm{c}} \sqrt{\frac{m_{\mathrm{c}} C}{W_{0}}}=\sqrt{\tilde{m}_{\mathrm{c}}}
$$

si $\gamma_{\mathrm{c}}^{2}$ est négatif, le point de fonctionnement est stable.

c) Système en vibrations forcées : réponse du palier à un balourd résiduel

Dans l'annexe (B), on montre que la trajectoire du centre de l'arbre prédite par l'approche linéaire est toujours de forme elliptique pour un balourd de faible excentricité. Celle-ci est obtenue à partir de la résolution du système d'équations différentielles suivant :

$$
\begin{aligned}
{\left[\begin{array}{cc}
\tilde{m} & 0 \\
0 & \tilde{m}
\end{array}\right]\left\{\begin{array}{c}
\tilde{X}^{\prime \prime} \\
\tilde{Y}^{\prime \prime}
\end{array}\right\}+\left\{\begin{array}{ll}
B_{X X} & B_{X Y} \\
B_{Y X} & B_{Y Y}
\end{array}\right\}\left\{\begin{array}{c}
\tilde{X}^{\prime} \\
\tilde{Y}^{\prime}
\end{array}\right\} } \\
+\left[\begin{array}{cc}
A_{X X} & A_{X Y} \\
A_{Y X} & A_{Y Y}
\end{array}\right]\left\{\begin{array}{c}
\tilde{X} \\
\tilde{Y}
\end{array}\right\}=\tilde{m} \varepsilon_{\mathrm{b}}\left\{\begin{array}{c}
\cos \tilde{t} \\
\sin \tilde{t}
\end{array}\right\}
\end{aligned}
$$

où $\tilde{m}=\frac{m \omega_{a}^{2} C}{W_{0}} ; A_{i j}=a_{i j} \frac{C}{W_{0}} ; B_{i j}=b_{i j} \frac{C \omega_{\mathrm{a}}}{W_{0}} ; \tilde{t}=\omega_{\mathrm{a}} t$; $\tilde{X}=\frac{X}{C} ; \tilde{Y}=\frac{Y}{C} ; \tilde{X}^{\prime}=\frac{\dot{X}}{C \omega_{\mathrm{a}}} ; \tilde{Y}^{\prime}=\frac{\dot{Y}}{C \omega_{\mathrm{a}}} ;\left(\tilde{X^{\prime \prime}}, \tilde{Y^{\prime \prime}}\right)=$ $\frac{(\ddot{X}, \ddot{Y})}{C \omega_{\mathrm{a}}^{2}} ; \varepsilon_{\mathrm{b}}=\frac{e_{\mathrm{b}}}{C}$.

\section{Résolution numérique}

Dans le cas d'un palier de longueur finie (écoulement bidimensionnel), on ne peut pas envisager une résolution analytique du système d'équations (22). La méthode de discrétisation retenue dans cette étude est celle des différences finies centrées. Le domaine de discrétisation est $(0,2 \pi) \times(-1 / 2,1 / 2)$. Les pas du maillage sont $\Delta \theta$ et $\Delta \tilde{z}$ tels que $\Delta \theta=\frac{2 \pi}{N_{\theta}}$ et $\Delta \tilde{z}=\frac{1}{N_{z}} . N_{\theta}$ et $N_{z}$ étant respectivement les nombres de mailles suivant $\theta$ et $\tilde{z}$.
Après discrétisation, le système d'équations (22) prend la forme suivante :

$$
\begin{array}{r}
\tilde{p}_{i j}^{(0)}=a_{i j} \tilde{p}_{i+1, j}^{(0)}+b_{i j} \tilde{p}_{i-1, j}^{(0)}+c_{i j} \tilde{p}_{i, j+1}^{(0)}+d_{i j} \tilde{p}_{i, j-1}^{(0)}-e_{i j}^{(0)} \\
\tilde{p}_{i j}^{(\varepsilon)}=a_{i j} \tilde{p}_{i+1, j}^{(\varepsilon)}+b_{i j} \tilde{p}_{i-1, j}^{(\varepsilon)}+c_{i j} \tilde{p}_{i, j+1}^{(\varepsilon)}+d_{i j} \tilde{p}_{i, j-1}^{(\varepsilon)}-e_{i j}^{(\varepsilon)} \\
\tilde{p}_{i j}^{(\phi)}=a_{i j} \tilde{p}_{i+1, j}^{(\phi)}+b_{i j} \tilde{p}_{i-1, j}^{(\phi)}+c_{i j} \tilde{p}_{i, j+1}^{(\phi)}+d_{i j} \tilde{p}_{i, j-1}^{(\phi)}-e_{i j}^{(\phi)}
\end{array}
$$

où les différentes matrices sont définies par :

$$
\begin{aligned}
& a_{i j}=\left(\frac{\tilde{G}_{0}}{\Delta \theta^{2}}+\frac{1}{2 \Delta \theta}\left(\frac{\partial \tilde{G}_{0}}{\partial \theta}\right)_{i j}\right) \frac{1}{F_{i j}} \\
& b_{i j}=\left(\frac{\tilde{G}_{0}}{\Delta \theta^{2}}-\frac{1}{2 \Delta \theta}\left(\frac{\partial \tilde{G}_{0}}{\partial \theta}\right)_{i j}\right) \frac{1}{F_{i j}} ; \\
& c_{i j}=\left(\frac{\tilde{G}_{0}}{\Delta \tilde{z}^{2}}+\frac{1}{2 \Delta \tilde{z}}\left(\frac{\partial \tilde{G}_{0}}{\partial \tilde{z}}\right)_{i j}\right) \frac{\lambda}{F_{i j}} ; \\
& d_{i j}=\left(\frac{\tilde{G}_{0}}{\Delta \tilde{z}^{2}}-\frac{1}{2 \Delta \tilde{z}}\left(\frac{\partial \tilde{G}_{0}}{\partial \tilde{z}}\right)_{i j}\right) \frac{\lambda}{F_{i j}} ; \\
& e_{i j}^{(0)}=6\left(\frac{\partial \tilde{h}_{0}}{\partial \theta}\right)_{i j} \frac{1}{F_{i j}} \text {; } \\
& F_{i j}=2 \tilde{G}_{0}\left(\frac{1}{\Delta \theta^{2}}+\frac{\lambda}{\Delta \tilde{z}^{2}}\right) \text {; } \\
& \lambda=\left(\frac{R}{L}\right)^{2} \\
& e_{i j}^{(\varepsilon)}=3 \frac{\tilde{h}_{0}^{2} \cos \theta}{\tilde{G}_{0}} e_{i j}^{(0)}+3 \tilde{G}_{0}\left(\frac{\partial \tilde{p}_{0}}{\partial \theta}\right)_{i j}\left(\frac{\partial f}{\partial \theta}\right)_{i j} \frac{1}{F_{i j}} \\
& +3 \lambda \tilde{G}_{0}\left(\frac{\partial \tilde{p}_{0}}{\partial \tilde{z}}\right)_{i j}\left(\frac{\partial f}{\partial \tilde{z}}\right)_{i j} \frac{1}{F_{i j}} \\
& +(6 \sin \theta-12 i \lambda \cos \theta) \frac{1}{F_{i j}} \\
& e_{i j}^{(\phi)}=3 \frac{\tilde{h}_{0}^{2} \sin \theta}{\tilde{G}_{0}} e_{i j}^{(0)}+3 \tilde{G}_{0}\left(\frac{\partial \tilde{p}_{0}}{\partial \theta}\right)_{i j}\left(\frac{\partial g}{\partial \theta}\right)_{i j} \frac{1}{F_{i j}} \\
& +3 \lambda \tilde{G}_{0}\left(\frac{\partial \tilde{p}_{0}}{\partial \tilde{z}}\right)_{i j}\left(\frac{\partial g}{\partial \tilde{z}}\right)_{i j} \frac{1}{F_{i j}} \\
& -(6 \cos \theta+12 i \lambda \sin \theta) \frac{1}{F_{i j}}
\end{aligned}
$$

Les différents gradients intervenant dans les relations cidessus sont évalués par les différences finies centrées.

Le système d'équations (38) est résolu numériquement à l'aide de la méthode itérative de Gauss-Seidel avec 

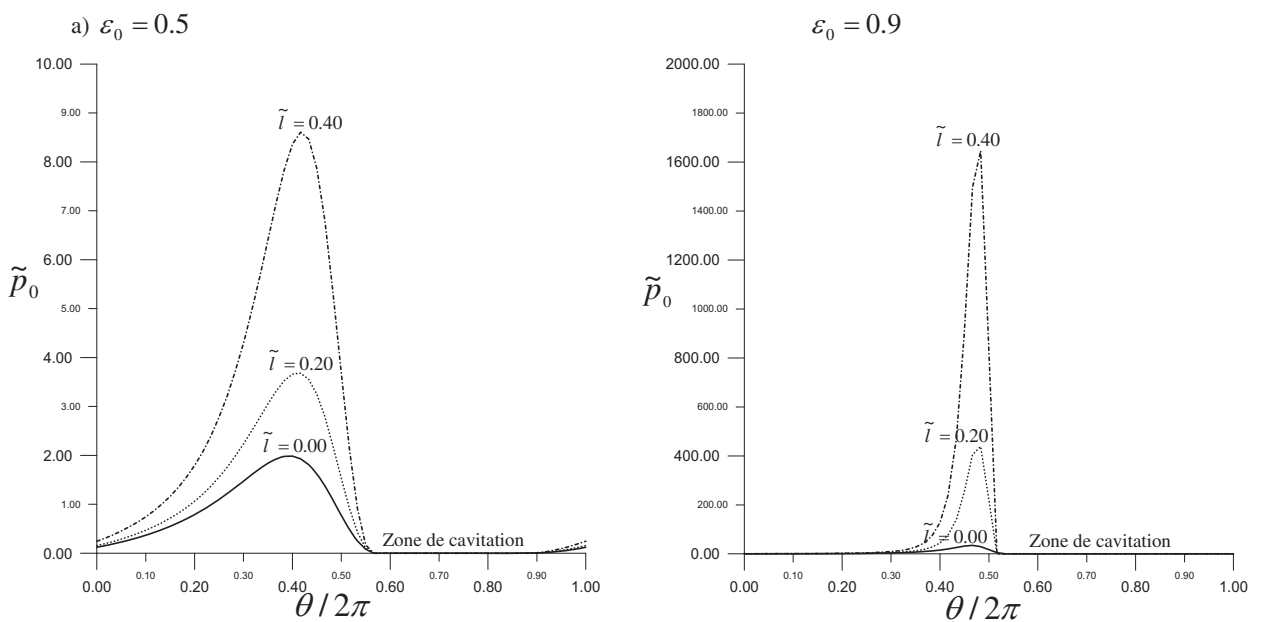

Fig. 4. Variations circonférentielles de la pression statique adimensionnée dans la section médiane du palier aligné pour différentes valeurs de $\tilde{l}\left(L / D=1, D_{\mathrm{m}}=0\right)$.

a) $\varepsilon_{0}=0.5$

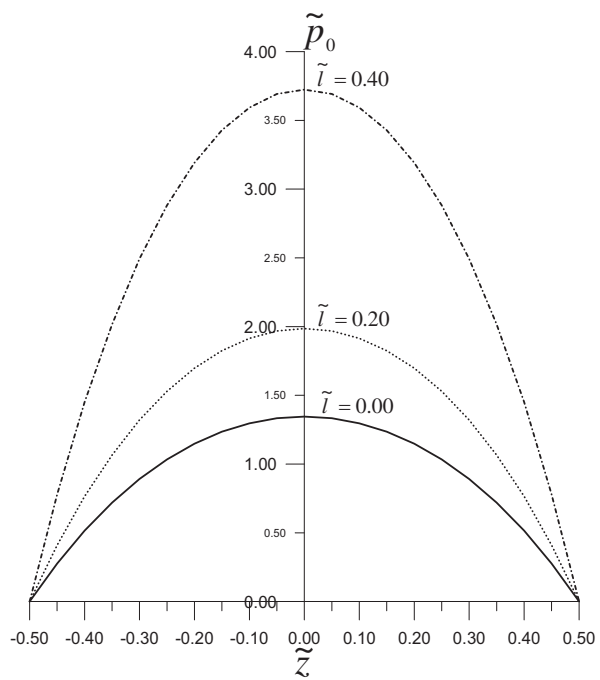

b) $\varepsilon_{0}=0.9$

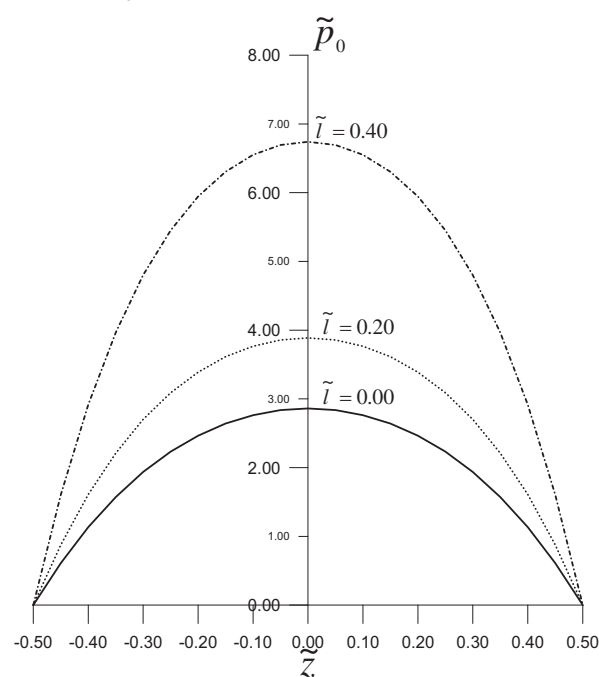

Fig. 5. Variations axiales de la pression statique adimensionnée dans une section longitudinale du palier aligné $\left(\theta=102^{\circ}\right)$ pour différentes valeurs de $\tilde{l}\left(L / D=1, D_{\mathrm{m}}=0\right)$.

coefficient de sur-relaxation. L'application de cet algorithme permet d'écrire :

$$
\begin{aligned}
\left(\tilde{p}_{i j}^{(0)}\right)^{k+1}= & (1-\Omega)\left(\tilde{p}_{i j}^{(0)}\right)^{k}+\Omega\left(a_{i j}\left(\tilde{p}_{i+1, j}^{(0)}\right)^{k}\right. \\
& +b_{i j}\left(\tilde{p}_{i-1, j}^{(0)}\right)^{k+1}+c_{i j}\left(\tilde{p}_{i, j+1}^{(0)}\right)^{k} \\
& \left.+d_{i j}\left(\tilde{p}_{i, j-1}^{(0)}\right)^{k+1}+e_{i j}^{(0)}\right) \\
\left(\tilde{p}_{i j}^{(\varepsilon)}\right)^{k+1}= & (1-\Omega)\left(\tilde{p}_{i j}^{(\varepsilon)}\right)^{k}+\Omega\left(a_{i j}\left(\tilde{p}_{i+1, j}^{(\varepsilon)}\right)^{k}\right. \\
& +b_{i j}\left(\tilde{p}_{i-1, j}^{(\varepsilon)}\right)^{k+1}+c_{i j}\left(\tilde{p}_{i, j+1}^{(\varepsilon)}\right)^{k} \\
& \left.+d_{i j}\left(\tilde{p}_{i, j-1}^{(\varepsilon)}\right)^{k+1}+e_{i j}^{(\varepsilon)}\right)
\end{aligned}
$$

$$
\begin{aligned}
\left(\tilde{p}_{i j}^{(\phi)}\right)^{k+1}= & (1-\Omega)\left(\tilde{p}_{i j}^{(\phi)}\right)^{k}+\Omega\left(a_{i j}\left(\tilde{p}_{i+1, j}^{(\phi)}\right)^{k}\right. \\
& +b_{i j}\left(\tilde{p}_{i-1, j}^{(\phi)}\right)^{k+1}+c_{i j}\left(\tilde{p}_{i, j+1}^{(\phi)}\right)^{k} \\
& \left.+d_{i j}\left(\tilde{p}_{i, j-1}^{(\phi)}\right)^{k+1}+e_{i j}^{(\phi)}\right)
\end{aligned}
$$

La valeur optimale de $\Omega$ permettant au processus itératif de converger en un nombre minimal d'itérations est déterminée par expérimentation numérique figure 3. 

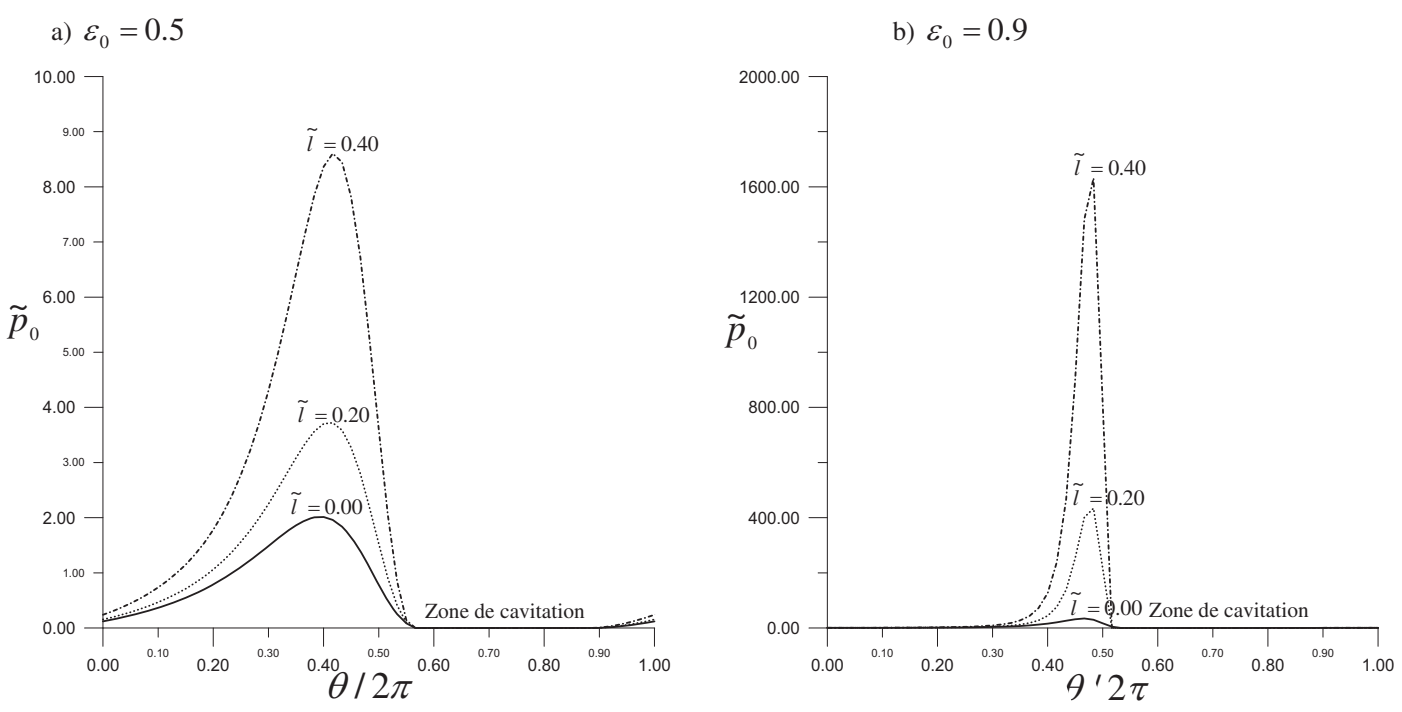

Fig. 6. Variations circonférentielles de la pression statique adimensionnée dans la section médiane du palier mésaligné pour différentes valeurs de $\tilde{l}\left(L / D=1, D_{\mathrm{m}}=0,5 \beta=0^{\circ}\right)$.

a) $\varepsilon_{0}=0.5$

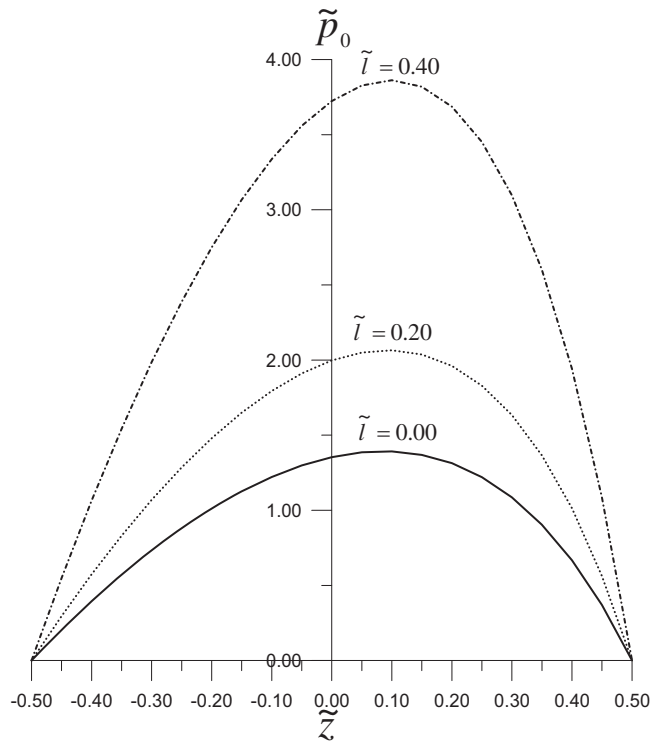

b) $\varepsilon_{0}=0.9$

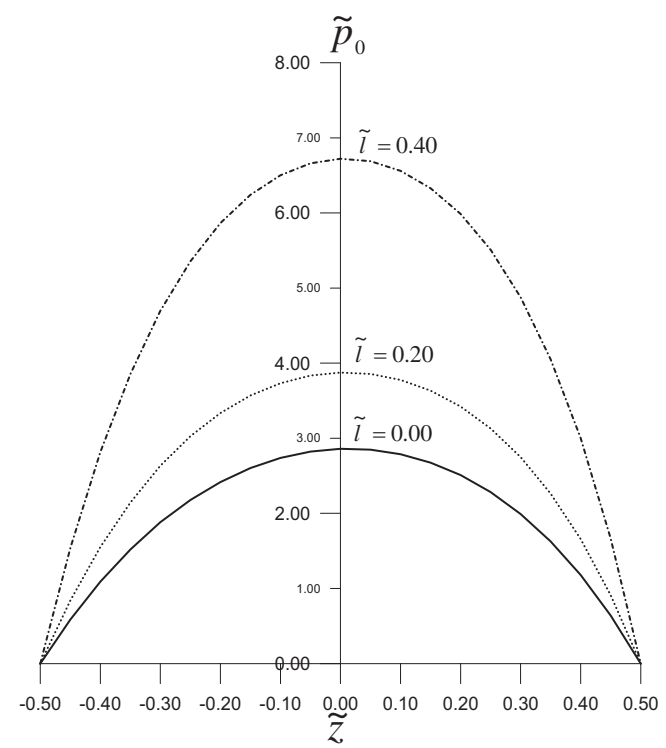

Fig. 7. Variations axiales de la pression statique adimensionnée dans une section longitudinale du palier mésaligné $\left(\theta=102^{\circ}\right)$ pour différentes valeurs de $\tilde{l}\left(L / D=1, D_{\mathrm{m}}=0,5, \beta=0^{\circ}\right)$.

Les tests d'arrêt des itérations sont :

$$
\begin{aligned}
& \max \left|\frac{\left(\tilde{p}_{i j}^{(0)}\right)^{k+1}-\left(\tilde{p}_{i j}^{(0)}\right)^{k}}{\left(\tilde{p}_{i j}^{(0)}\right)^{k+1}}\right| \leqslant 0,00001 ; \\
& \max \left|\frac{\left(\tilde{p}_{i j}^{(\varepsilon)}\right)^{k+1}-\left(\tilde{p}_{i j}^{(\varepsilon)}\right)^{k}}{\left(\tilde{p}_{i j}^{(\varepsilon)}\right)^{k+1}}\right| \leqslant 0,00001 ; \\
& \max \left|\frac{\left(\tilde{p}_{i j}^{(\phi)}\right)^{k+1}-\left(\tilde{p}_{i j}^{(\phi)}\right)^{k}}{\left(\tilde{p}_{i j}^{(\phi)}\right)^{k+1}}\right| \leqslant 0,00001
\end{aligned}
$$

où $k$ est le numéro de l'itération.

Les différentes intégrales intervenant dans les expressions des caractéristiques statiques et dynamiques sont évaluées numériquement à l'aide de la formule des trapèzes généralisée.

\section{Résultats et discussion}

Afin d'étudier les effets du paramètre du couple de contrainte sur les performances statiques et dynamiques de paliers lisses de longueur finie $(L / D=1)$ dans le cas aligné $\left(D_{\mathrm{m}}=0,00\right)$ et mésaligné $\left(D_{\mathrm{m}}=0,50\right)$. Les calculs 
a) $\tilde{l}=0.00$

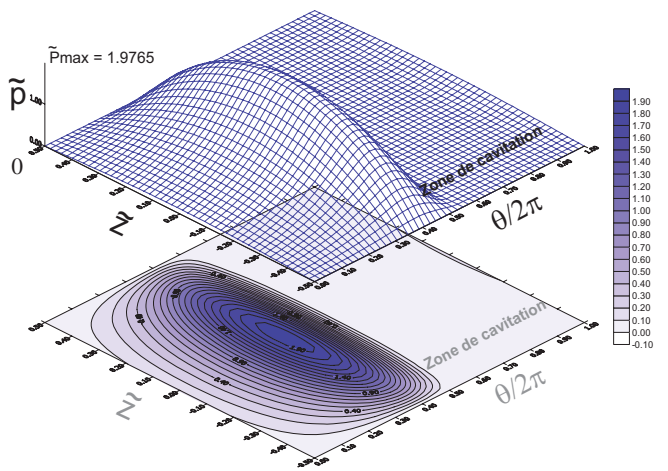

b) $\tilde{l}=0.20$

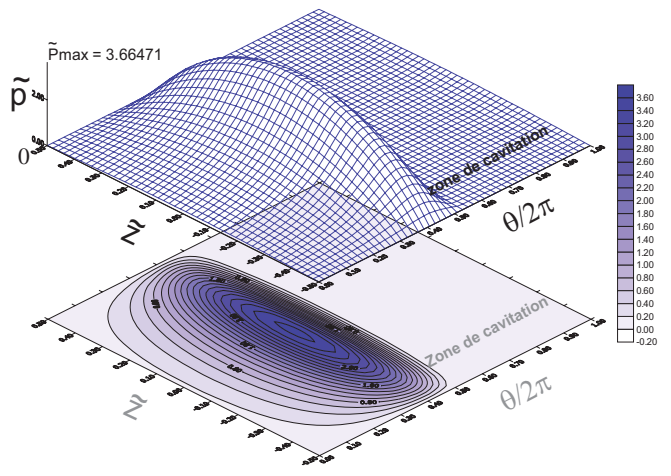

c) $\tilde{l}=0.40$

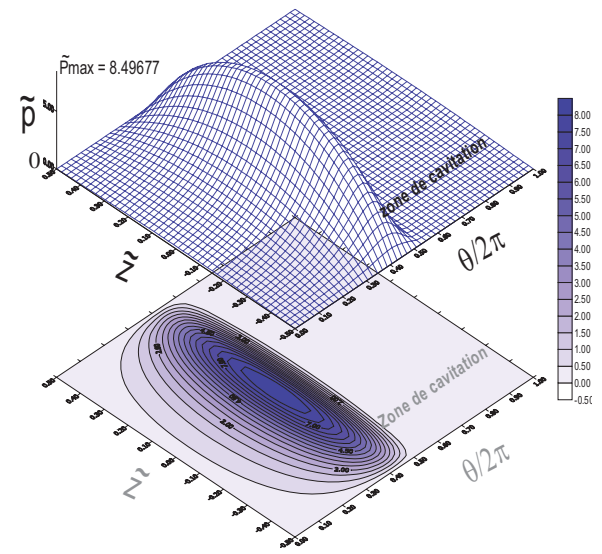

Fig. 8. Distributions de la pression statique dans un palier aligné faiblement chargé pour différentes valeurs de $\tilde{l}\left(\varepsilon_{0}=\right.$ $0,5)$.

ont été faits pour trois valeurs du paramètre de couple des contraintes $\tilde{l}=0,00$ (fluide newtonien), 0,20 et 0,40 (fluide polaire non newtonien).

\subsection{Champ de pression hydrodynamique statique}

Les distributions circonférentielle et axiale du champ de pression statique sont déterminées pour deux valeurs a) $\tilde{l}=0.00$

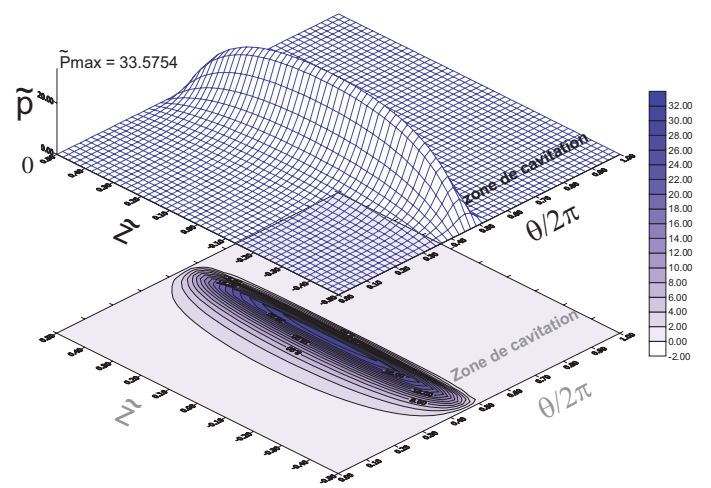

b) $\tilde{l}=0.20$

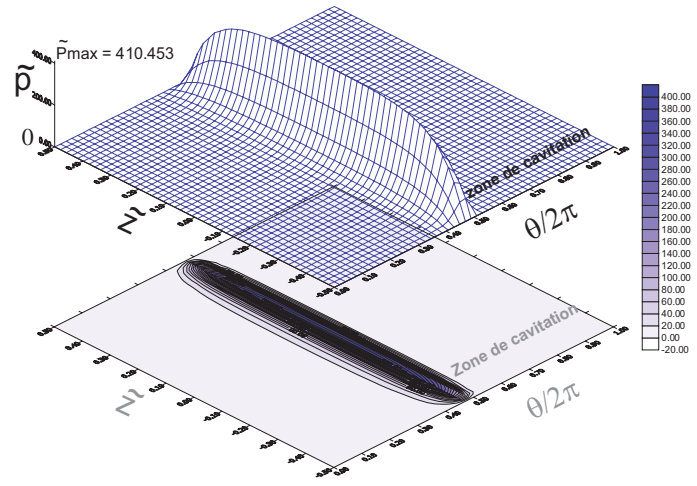

c) $\tilde{l}=0.40$

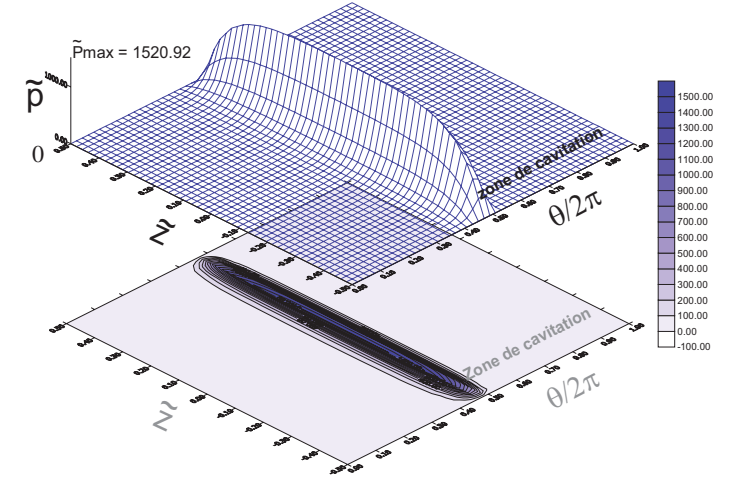

Fig. 9. Distributions de la pression statique dans un palier aligné lourdement chargé pour différentes valeurs de $\tilde{l}\left(\varepsilon_{0}=\right.$ $0,9)$.

de l'excentricité relative statique $\varepsilon_{0}=0,50$ et 0,90 , pour $\theta=102^{\circ}$ et $\tilde{z}=0$ (section médiane du palier). Les représentations graphiques bidimensionnelles et tridimensionnelles (Figs. 4 à 11) montrent que la pression statique maximale augmente avec le paramètre du couple de contrainte ou la longueur de la chaîne moléculaire; cette augmentation est d'autant plus importante que l'excentricité relative statique de fonctionnement est grande. On remarque aussi que les pressions maximales obtenues pour le palier mésaligné sont supérieures à celles du palier 
a) $\tilde{l}=0.00$

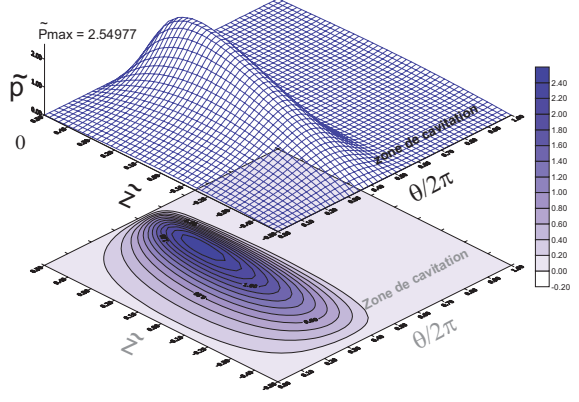

b) $\tilde{l}=0.20$

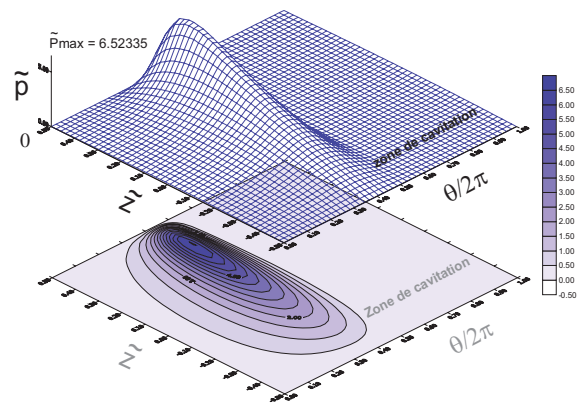

c) $\tilde{l}=0.40$

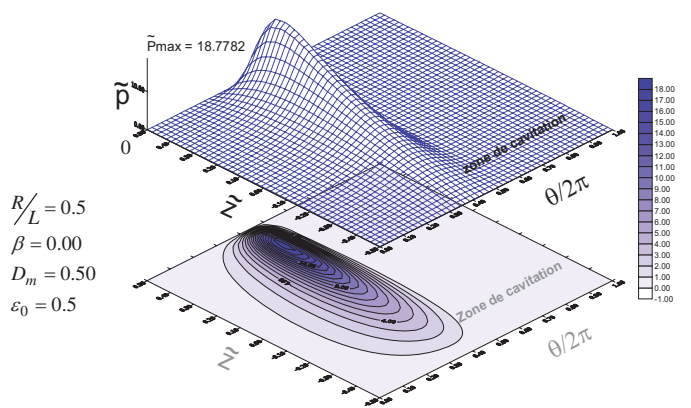

Fig. 10. Distributions de la pression statique dans un palier mésaligné faiblement chargé pour différentes valeurs de $\tilde{l}\left(\varepsilon_{0}=\right.$ $\left.0,5, D_{\mathrm{m}}=0,5, \beta=0^{\circ}\right)$.

aligné. Il est aussi important de souligner que la présence du mésalignement entraîne une répartition dissymétrique de la pression et un déplacement du pic de pression vers l'extrémité du palier.

\subsection{Performances statiques}

Les figures 12 à 14 représentent les variations de la portance hydrodynamique adimensionnée, de l'angle de calage et du nombre de frottement en fonction de l'excentricité relative statique. Les résultats obtenus montrent:

- une augmentation de la portance hydrodynamique avec le paramètre du couple de contrainte; cette augmentation est plus significative à forte excentricité c'est-à-dire lorsque le palier est lourdement chargé. On remarque aussi que les valeurs obtenues pour le palier mésaligné sont supérieures à celles du palier aligné; a) $\tilde{l}=0.00$

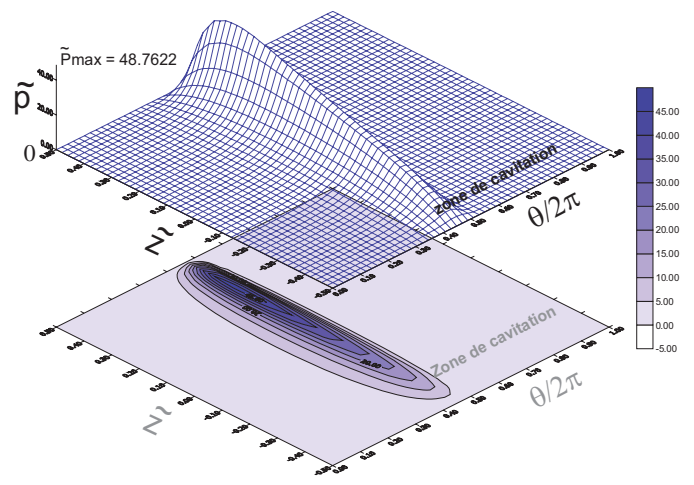

b) $\tilde{l}=0.20$

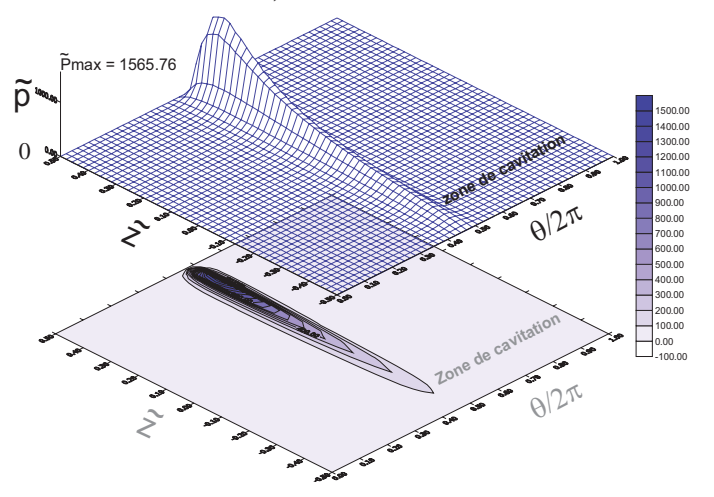

c) $\tilde{l}=0.40$

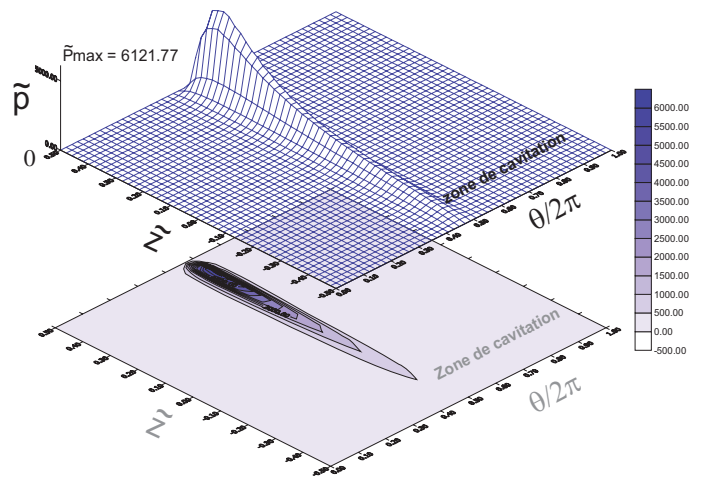

Fig. 11. Distributions de la pression statique dans un palier mésaligné lourdement chargé pour différentes valeurs de $\tilde{l}$ $\left(\varepsilon_{0}=0,9, D_{\mathrm{m}}=0,5, \beta=0^{\circ}\right)$.

- une diminution de l'angle de calage aussi bien pour le palier aligné que le palier mésaligné, cette diminution est plus importante pour les valeurs élevées du paramètre de couple de contrainte;

- une diminution du nombre de frottement avec le paramètre de couple de contrainte cette diminution est plus significative lorsque le palier est lourdement chargé. On remarque aussi que les valeurs obtenues pour le palier mésaligné sont légèrement inférieures à celles du palier aligné. 
a) $\mathrm{D}_{\mathrm{m}}=0.00$

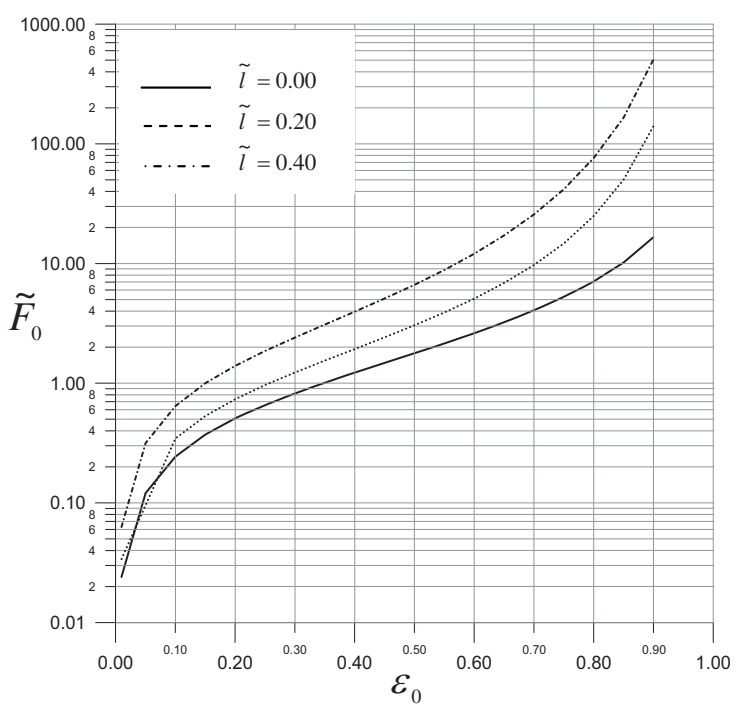

b) $\mathrm{D}_{\mathrm{m}}=0.50, \beta=0^{\circ}$

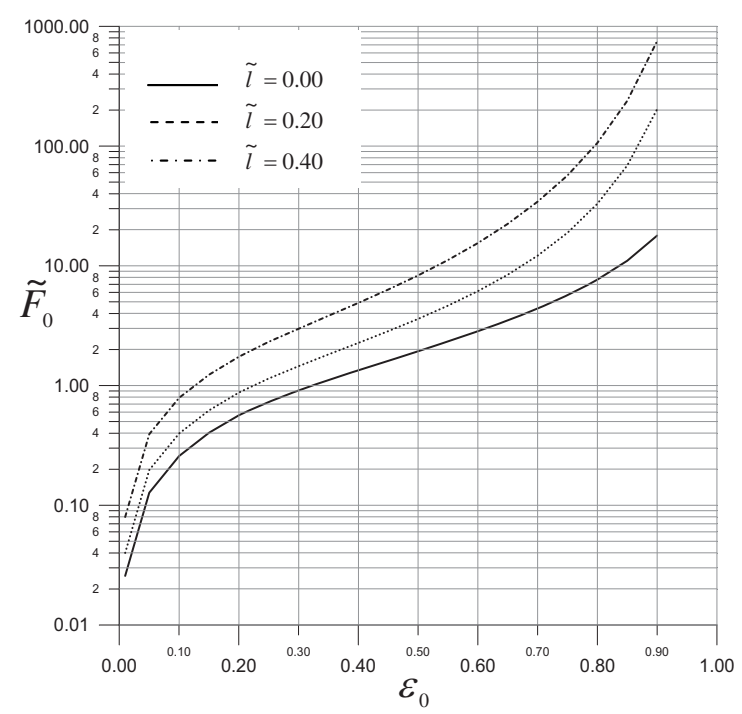

Fig. 12. Variations de la portance hydrodynamique adimensionnée en fonction de l'excentricité relative statique pour différentes valeurs de $\tilde{l}$.
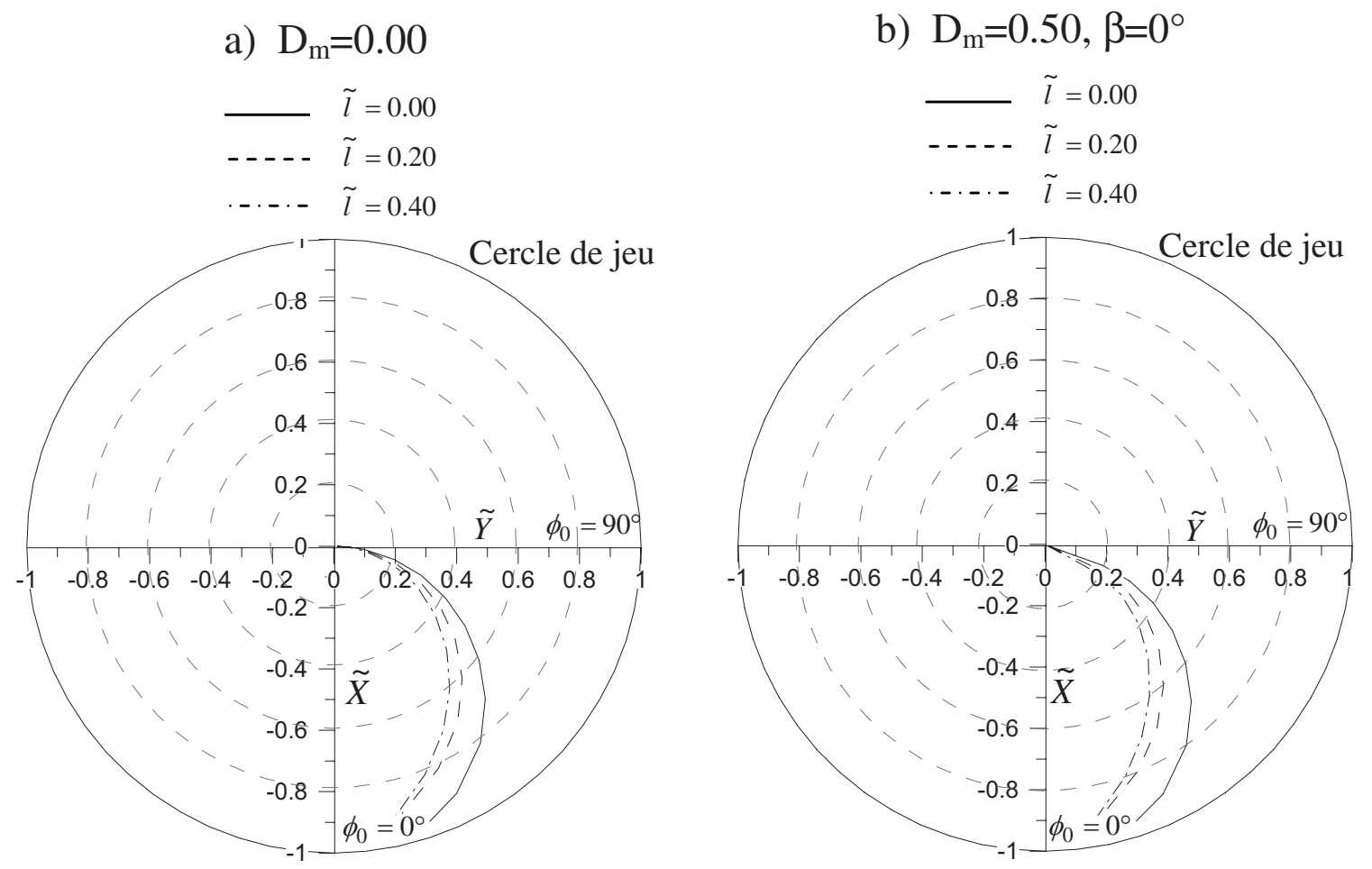

Fig. 13. Variations de l'angle de calage en fonction de l'excentricité relative statique pour différentes valeurs de $\tilde{l}$.

On peut conclure que la présence des additifs de viscosité dans les huiles lubrifiantes permet d'améliorer la capacité de charge du palier et de réduire les pertes par frottement dans le contact surtout pour les additifs de longues chaînes moléculaires.

La figure 15 représente les variations du débit de fuite moyen en fonction de l'excentricité relative statique. Les résultats obtenus pour les deux paliers alignés et mésalignés montrent que la rhéologie du lubrifiant n'a pas d'effets appréciables sur le débit sauf pour les grandes valeurs de l'excentricité relative.

La figure 16 représente les variations du moment de mésalignement en fonction de l'excentricité relative statique pour deux valeurs du degré de mésalignement $D_{\mathrm{m}}=$ $20 \%$ et $50 \%$. Pour une excentricité donnée, les résultats obtenus montrent que le moment de mésalignement augmente avec le paramètre du couple de contrainte et le degré de mésalignement. On peut conclure que la 
a) $\mathrm{D}_{\mathrm{m}}=0.00$

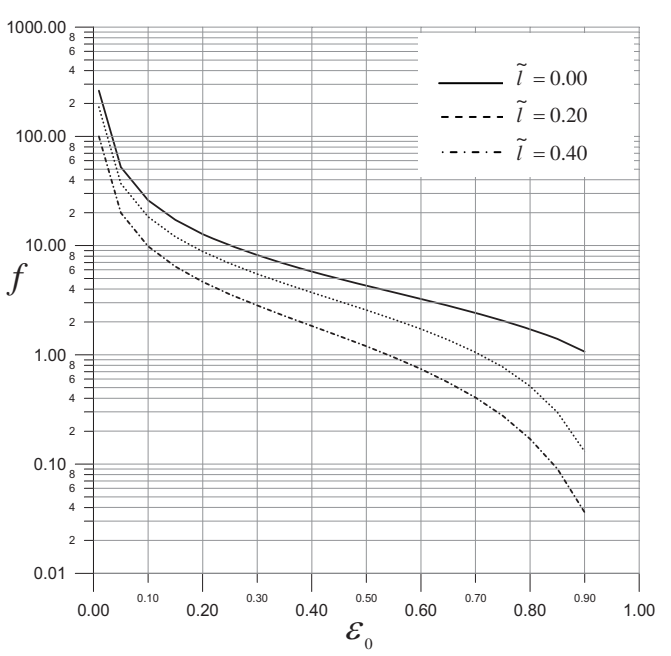

b) $\mathrm{D}_{\mathrm{m}}=0.50, \beta=0^{\circ}$

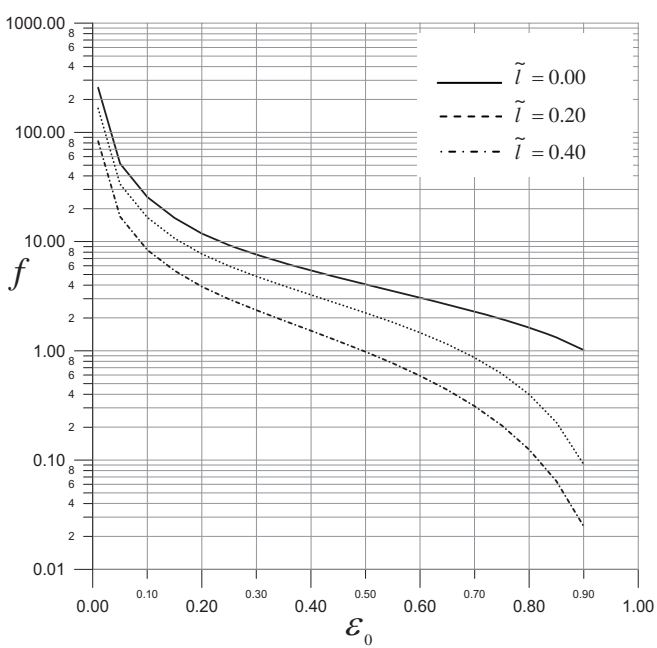

Fig. 14. Variations du nombre de frottement en fonction de l'excentricité relative statique pour différentes valeurs de $\tilde{l}$.

a) $\mathrm{D}_{\mathrm{m}}=0.00$

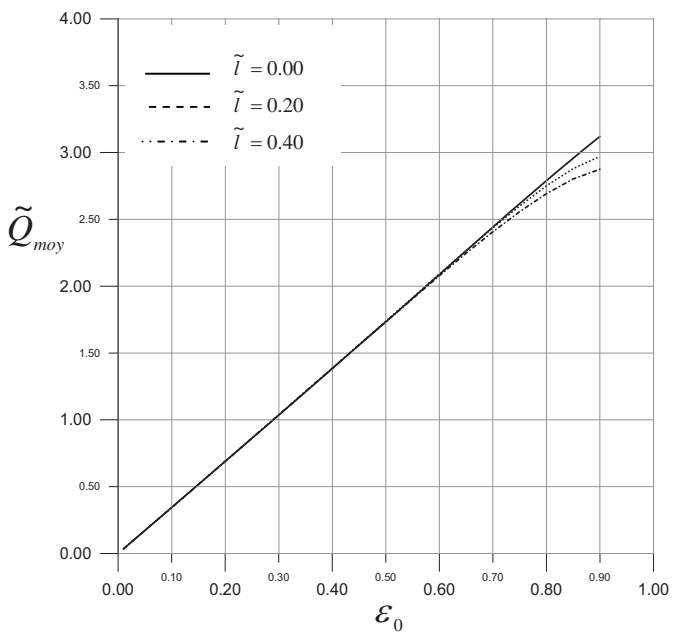

b) $\mathrm{D}_{\mathrm{m}}=0.50, \beta=0^{\circ}$

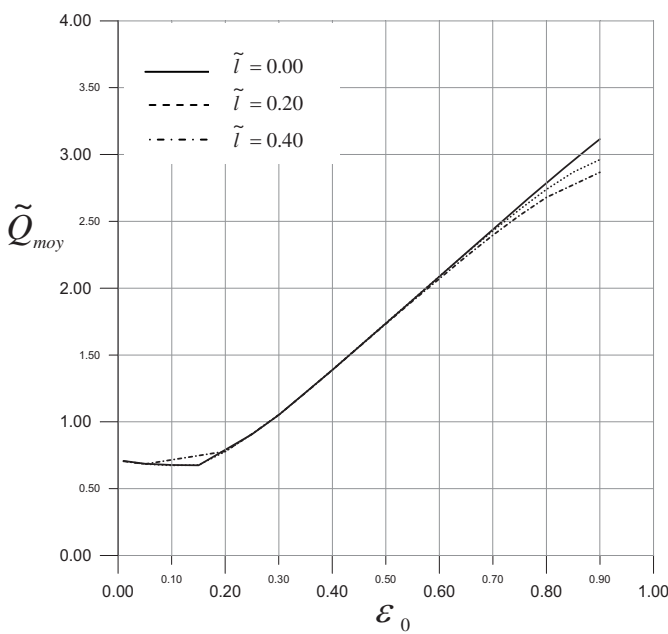

Fig. 15. Variations du débit de fuite moyen adimensionné en fonction de l'excentricité relative statique pour différentes valeurs de $\tilde{l}$.

a) $D_{m}=0.20, \beta=0^{\circ}$

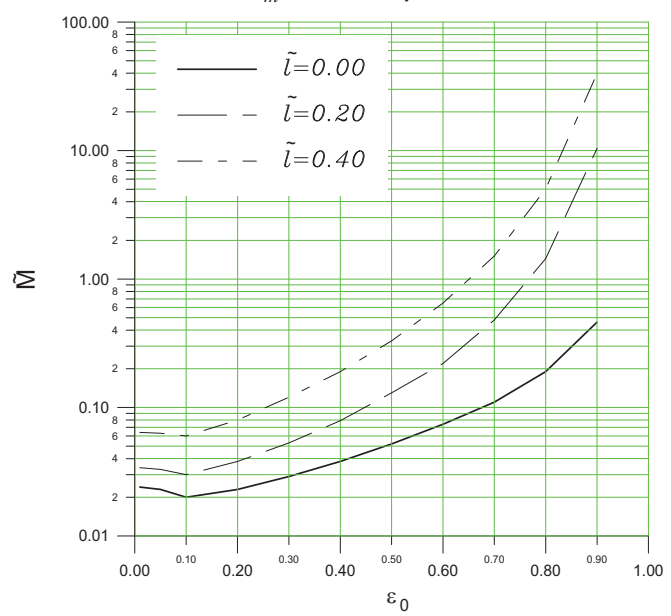

b) $D_{m}=0.50, \beta=0^{\circ}$

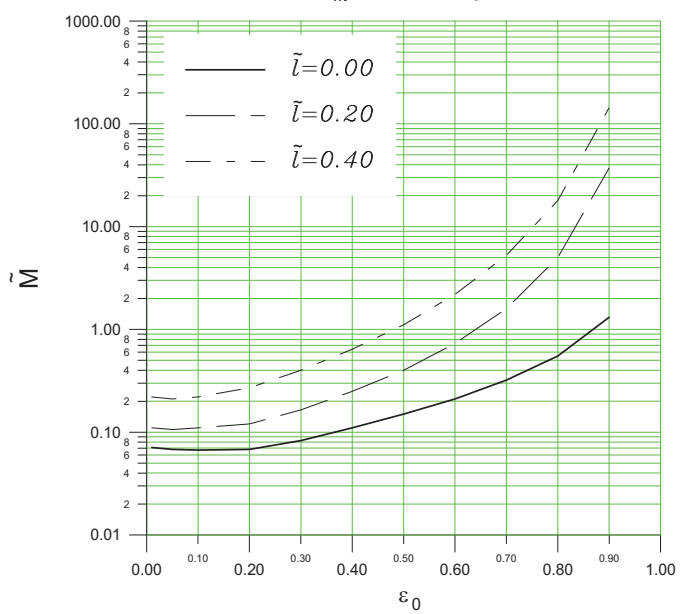

Fig. 16. Variations du moment de mésalignement adimensionné en fonction de l'excentricité relative statique pour différentes valeurs de $l$. 
a) $\mathrm{D}_{\mathrm{m}}=0.00$

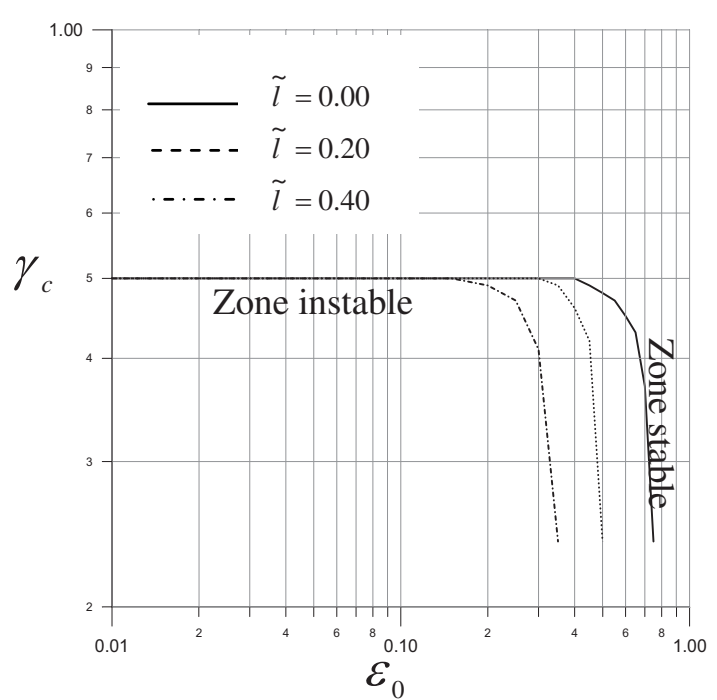

b) $\mathrm{D}_{\mathrm{m}}=0.50, \beta=0^{\circ}$

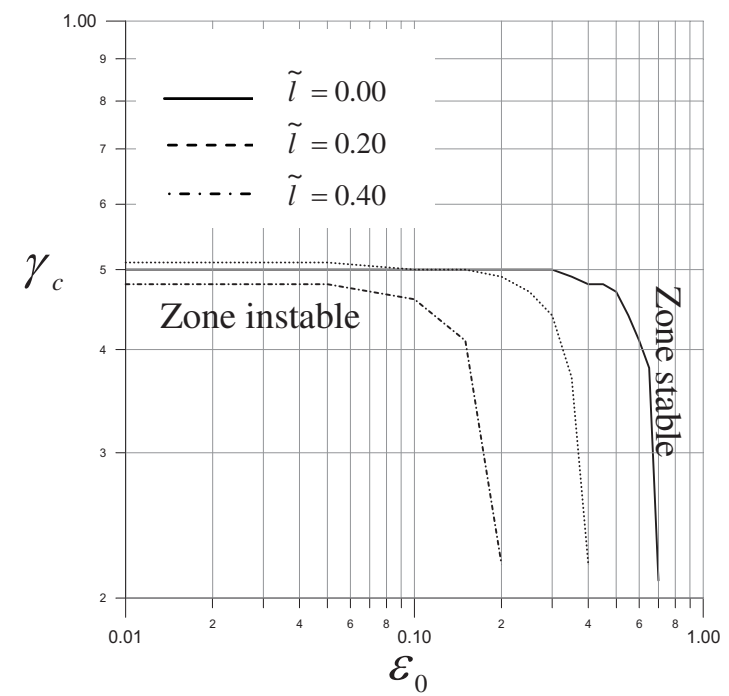

Fig. 17. Variations de la pulsation de fouettement (whirl) en fonction de l'excentricité relative statique pour différentes valeurs de $l$.

a) $\mathrm{D}_{\mathrm{m}}=0.00$

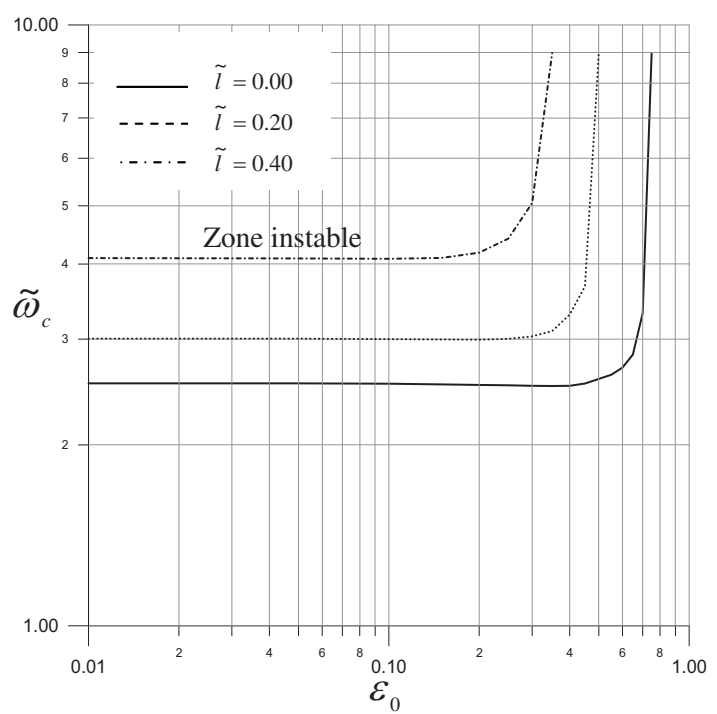

b) $\mathrm{D}_{\mathrm{m}}=0.50, \beta=0^{\circ}$

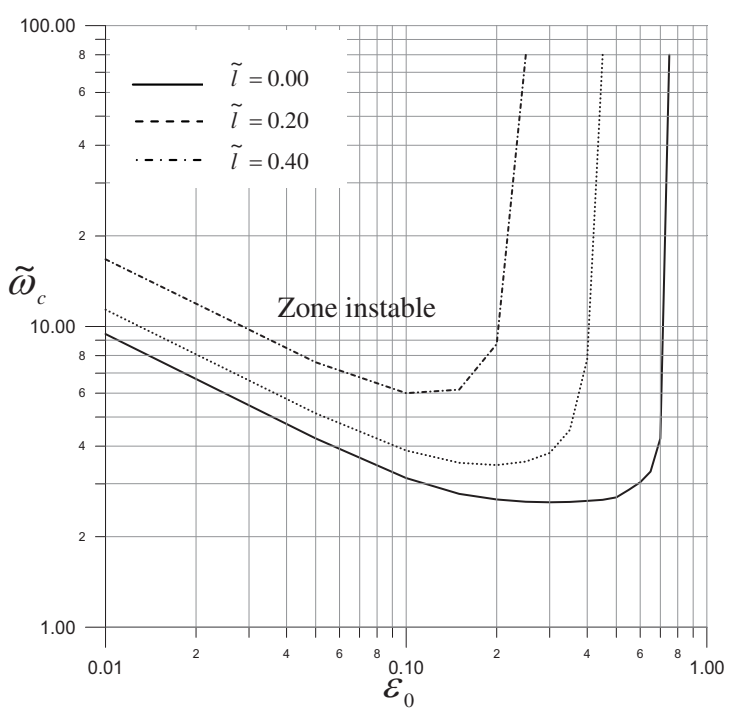

Fig. 18. Variations de la vitesse critique en fonction de l'excentricité relative statique pour différentes valeurs de $\tilde{l}$.

Tableau 1. Caractéristiques géométriques des trajectoires du centre de l'arbre pour différentes valeurs de $\tilde{l}$.

\begin{tabular}{lllll}
\hline$\tilde{l}$ & $\tilde{a}$ & $\tilde{b}$ & $\delta^{*}(\mathrm{deg})$ & $\varphi(\mathrm{deg})$ \\
\hline 0,0 (cas newtonien) & 0,12 & 0,02 & 68 & 38 \\
0,2 (cas polaire) & 0,02 & 0,0002 & 83 & 80 \\
0,4 (cas polaire) & 0,005 & 0,00005 & 85 & 84 \\
\hline
\end{tabular}

présence des additifs de viscosité dans les huiles lubrifiantes entraîne une augmentation de ce moment, cette augmentation est d'autant plus grande que le degré de mésalignement est important. Ceci est en parfait accord avec la dissymétrie du champ de pression représenté figures 10 et 11 .

Les figures 17 et 18 représentent les cartes de stabilité des paliers aligné et mésaligné. On remarque que la présence des additifs de viscosité dans les huiles lubrifiantes a un effet positif vis-à-vis de la stabilité du palier. En effet, on remarque une réduction de la zone d'instabilité et une augmentation de la vitesse critique avec le paramètre du couple de contrainte. Par comparaison aux fluides newtoniens (huiles non additivées), les huiles contenant des polymères de longues chaînes moléculaires permettent de rendre le palier stable même aux faibles valeurs de l'excentricité de fonctionnement car il est bien 
connu que ce type de paliers constitue une source de vibrations pour la ligne d'arbre qu'ils supportent lorsqu'ils sont faiblement chargés et lubrifiés par des huiles newtoniennes.

Dans la figure 19, on présente les orbites du centre de l'arbre calculées pour une excentricité relative du balourd égale à 0,15 , une masse adimensionnée égale à $\tilde{m}=\frac{C \omega_{\mathrm{a}}^{2}}{g}=0,5$ et différentes valeurs du paramètre du couple de contrainte. Ces orbites ont été obtenues à partir de la résolution analytique des équations de mouvement du rotor rigide (37). On observe une atténuation significative des amplitudes de vibration du centre de l'arbre avec le paramètre du couple de contrainte. Les valeurs numériques des caractéristiques géométriques de ces orbites sont reportées dans le tableau 1 pour les trois valeurs du paramètre du couple de contrainte.

\section{Conclusions}

L'étude paramétrique a montré que la présence des additifs de viscosité dans les lubrifiants a une influence non négligeable sur le comportement statique et dynamique des paliers lisses surtout pour les grandes valeurs du paramètre du couple de contrainte. Toutefois, leur influence sur le débit de fuite est négligeable sauf pour les grandes excentricités de fonctionnement. Par comparaison aux huiles newtoniennes, les huiles additivées permettent :

- une augmentation importante de la portance hydrodynamique et du pic de la pression statique dans le film lubrifiant surtout dans le cas du palier mésaligné;

- une diminution de l'angle de calage et des pertes par frottement dans le contact;

- une augmentation de la vitesse critique;

- une réduction de la zone d'instabilité ce qui permet d'éviter le phénomène de fouettement (whirl) même lorsque le palier est modérément chargé;

- une atténuation significative des amplitudes de vibrations dues à la présence d'un balourd;

- par comparaison au cas aligné, le cas mésaligné présente pour une même valeur d'excentricité :

- une augmentation de la capacité de charge du palier et de l'angle de calage;

- une réduction du nombre de frottement et de la zone de stabilité.

En outre, le palier mésaligné possède un meilleur comportement statique mais une stabilité moindre comparée au cas du palier aligné.

\section{Annexe A}

\section{Expression des composantes de la vitesse de la surface de l'arbre}

Soient $U_{2}$ et $V_{2}$ les composantes (suivant les directions $x$ et $y$ ) du vecteur vitesse $\vec{V}_{2}$ d'un point $M_{2}$ appartenant

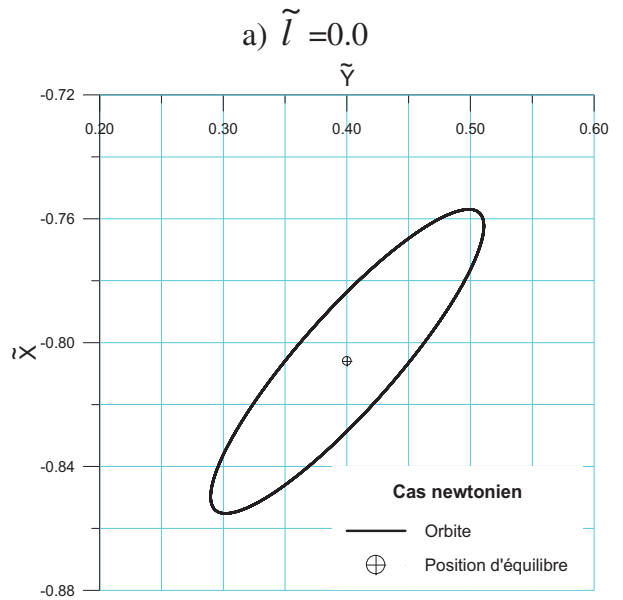

b) $\tilde{l}=0.2$

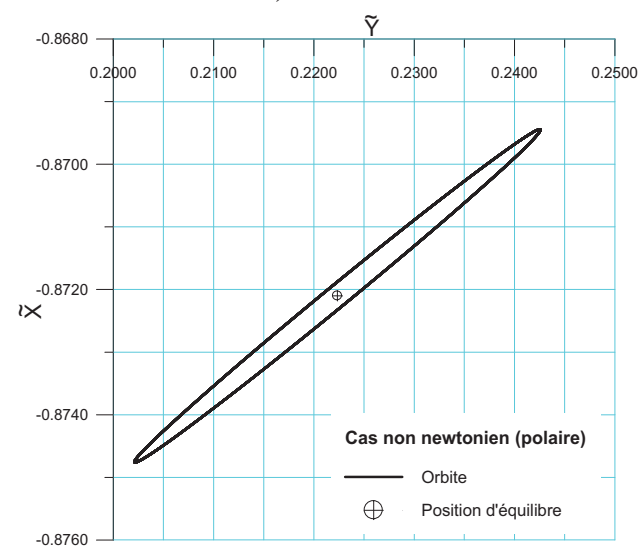

c) $\tilde{l}=0.4$

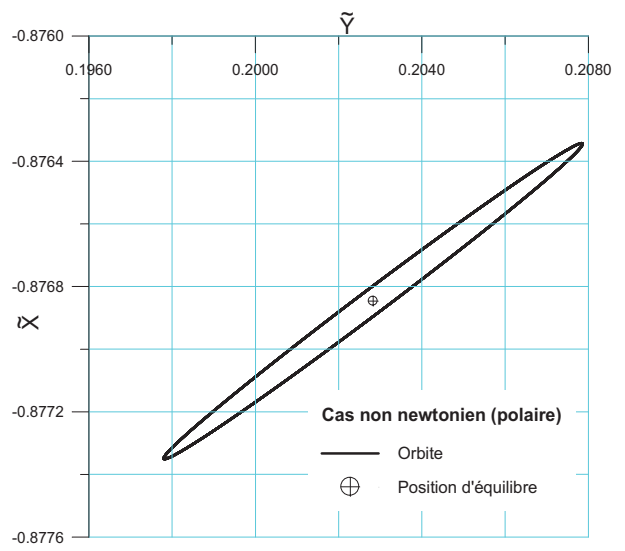

Fig. 19. Trajectoires du centre de l'arbre calculées pour $\varepsilon_{0}=$ $0,9, D_{\mathrm{m}}=0,0$ et différentes valeurs de $\tilde{l}$.

à la surface de l'arbre $\left(S_{2}\right)$ (Fig. 1), tel que :

$$
\overrightarrow{V_{2}}=\vec{V}\left(M_{2}\right)=\vec{V}\left(O_{\mathrm{a}}\right)+\overrightarrow{M_{2} O_{\mathrm{a}}} \Lambda \vec{\omega} \mathrm{a}
$$

où $\vec{V}\left(O_{\mathrm{a}}\right)$ est la vitesse du centre de l'arbre et $\vec{\omega}_{\mathrm{a}}$ le vecteur rotation.

Dans le repère $(\vec{\varepsilon}, \vec{\phi}, \vec{z})$, on a :

$$
\vec{V}\left(O_{\mathrm{a}}\right)=\dot{e} \vec{\varepsilon}+e \dot{\phi} \vec{\phi}=C \dot{\varepsilon} \vec{\varepsilon}+C \varepsilon \dot{\phi} \vec{\phi}
$$


Dans le repère $\left(\vec{n}_{2}, \vec{s}_{2}, \vec{z}\right)$, on peut écrire :

$$
\overrightarrow{M_{2} \mathrm{O}_{\mathrm{a}}}=-R \vec{n}_{2}
$$

Expression de $(\vec{\varepsilon}, \vec{\phi}, \vec{z})$ en fonction de $\left(\vec{n}_{2}, \vec{s}_{2}, \vec{z}\right)$

$$
\begin{aligned}
& \vec{\varepsilon}=-\vec{n}_{2} \cos (\theta-\alpha)+\vec{s}_{2} \sin (\theta-\alpha) \\
& \vec{\phi}=-\vec{n}_{2} \cos \left(\frac{\pi}{2}-(\theta-\alpha)\right)-\vec{s}_{2} \sin \left(\frac{\pi}{2}-(\theta-\alpha)\right)
\end{aligned}
$$

Puisque $\alpha=\left|\frac{\partial h}{\partial x}\right|<<1$, on peut écrire :

$$
\begin{aligned}
& \vec{r}=-\vec{n}_{2} \cos \theta+\vec{s}_{2} \sin \theta \\
& \vec{t}=-\vec{n}_{2} \sin \theta-\vec{s}_{2} \cos \theta
\end{aligned}
$$

ou encore :

$$
\left\{\begin{array}{l}
\vec{\varepsilon} \\
\vec{\phi}
\end{array}\right\}=\left[\begin{array}{l}
-\cos \theta \sin \theta \\
-\sin \theta-\cos \theta
\end{array}\right]\left\{\begin{array}{l}
\vec{n}_{2} \\
\vec{s}_{2}
\end{array}\right\}
$$

la vitesse de $O_{\text {a }}$ dans le repère $\left(\vec{n}_{2}, \vec{s}_{2}, \vec{z}\right)$ est :

$$
\begin{aligned}
\vec{V}\left(O_{\mathrm{a}}\right)= & C \dot{\varepsilon}\left(-\vec{n}_{2} \cos \theta+\vec{s}_{2} \sin \theta\right) \\
& +C \varepsilon \dot{\phi}\left(-\vec{n}_{2} \sin \theta-\vec{s}_{2} \cos \theta\right) \\
\vec{V}\left(O_{\mathrm{a}}\right)= & \vec{n}_{2}(-C \dot{\varepsilon} \cos \theta-C \varepsilon \dot{\phi} \sin \theta) \\
& +\vec{s}_{2}(C \dot{\varepsilon} \sin \theta-C \varepsilon \dot{\phi} \cos \theta)
\end{aligned}
$$

Expression de $\left(\vec{n}_{2}, \vec{s}_{2}, \vec{z}\right)$ en fonction de $(\vec{i}, \vec{j}, \vec{k})$

$$
\left\{\begin{array}{l}
\vec{n}_{2} \\
\vec{s}_{2}
\end{array}\right\}=\left[\begin{array}{l}
\sin \alpha-\cos \alpha \\
\cos \alpha \sin \alpha
\end{array}\right]\left\{\begin{array}{l}
\vec{i} \\
\vec{j}
\end{array}\right\}
$$

on a alors :

$$
\overrightarrow{M_{2} O_{\mathrm{a}}}=-R \overrightarrow{n_{2}}=-R \vec{i} \sin \alpha+R \vec{j} \cos \alpha
$$

et

$$
\begin{aligned}
\overrightarrow{M_{2} O_{\mathrm{a}}} \Lambda \overrightarrow{\omega_{\mathrm{a}}}= & \left|\begin{array}{lll}
\vec{i} & \vec{j} & \vec{k} \\
-R \sin \alpha & R \cos \alpha & 0 \\
0 & 0 & \omega_{\mathrm{a}}
\end{array}\right| \\
\quad & =\vec{i} \omega_{\mathrm{a}} R \cos \alpha-\vec{j} R \omega_{\mathrm{a}} \sin \alpha+\vec{k} 0
\end{aligned}
$$

soit

$$
\overrightarrow{M_{2} O_{\mathrm{a}}} \Lambda \overrightarrow{\omega_{\mathrm{a}}}=R \omega_{\mathrm{a}}(\cos \alpha \vec{i}+\sin \alpha \vec{j})
$$

En utilisant la matrice de passage (A4), la vitesse du centre de l'arbre $O_{\mathrm{a}}$ dans le repère $(x, y, z)$ est :

$$
\begin{aligned}
\vec{V}\left(O_{\mathrm{a}}\right)= & (\sin \alpha \vec{i}-\cos \alpha \vec{j})(-C \dot{\varepsilon} \cos \theta-C \varepsilon \dot{\phi} \sin \theta) \\
& +(\cos \alpha \vec{i}+\sin \alpha \vec{j})(C \dot{\varepsilon} \sin \theta-C \varepsilon \dot{\phi} \cos \theta)
\end{aligned}
$$

Comme $\alpha$ est très petit, la vitesse du point $M_{2}$ dans le repère $(x, y, z)$ est :

$$
\begin{aligned}
& \vec{V}\left(M_{2}\right)=\vec{i}(-C \dot{\varepsilon} \cos \theta \frac{\partial h}{\partial x}-C \varepsilon \dot{\phi} \sin \theta \frac{\partial h}{\partial x} \\
&\left.+C \dot{\varepsilon} \sin \theta-C \varepsilon \dot{\phi} \cos \theta+R \omega_{\mathrm{a}}\right) \\
&+\vec{j}\left(+C \dot{\varepsilon} \cos \theta+C \varepsilon \dot{\phi} \sin \theta+C \dot{\varepsilon} \sin \theta \frac{\partial h}{\partial x}\right. \\
&\left.-C \varepsilon \dot{\phi} \sin \theta \frac{\partial h}{\partial x}+R \omega_{\mathrm{a}} \frac{\partial h}{\partial x}\right)
\end{aligned}
$$

d'où les expressions des composantes du vecteur vitesse $\vec{V}_{2}$ suivant les directions $\vec{i}$ et $\vec{j}$ :

$$
\begin{aligned}
U_{2}= & \omega_{\mathrm{a}} R+C \dot{\varepsilon} \sin \theta-C \varepsilon \dot{\phi} \cos \theta \\
& -\frac{\partial h}{\partial x}(+C \dot{\varepsilon} \cos \theta+C \varepsilon \dot{\phi} \sin \theta) \\
V_{2}= & \omega_{\mathrm{a}} R \frac{\partial h}{\partial x}+C \dot{\varepsilon} \cos \theta+C \varepsilon \dot{\phi} \sin \theta \\
& +\frac{\partial h}{\partial x}(C \dot{\varepsilon} \sin \theta-C \varepsilon \dot{\phi} \cos \theta)
\end{aligned}
$$

\section{Annexe B}

\section{Réponse d'un palier hydrodynamique à une excitation synchrone de type balourd}

Dans le cas de vibrations de faible amplitude de l'arbre au voisinage de la position d'équilibre $O_{\mathrm{a} 0}$ avec un balourd de faible excentricité (résiduel), les équations de mouvement du rotor rigide s'écrivent :

$$
\begin{aligned}
{\left[\begin{array}{cc}
m & 0 \\
0 & m
\end{array}\right]\left\{\begin{array}{l}
\ddot{X} \\
\ddot{Y}
\end{array}\right\}+\left\{\begin{array}{ll}
b_{X X} & b_{X Y} \\
b_{Y X} & b_{Y Y}
\end{array}\right\}\left\{\begin{array}{l}
\dot{X} \\
\dot{Y}
\end{array}\right\} } \\
+\left[\begin{array}{ll}
a_{X X} & a_{X Y} \\
a_{Y X} & a_{Y Y}
\end{array}\right]\left\{\begin{array}{c}
X \\
Y
\end{array}\right\}=m e_{\mathrm{b}} \omega_{\mathrm{a}}^{2}\left\{\begin{array}{c}
\cos \omega_{\mathrm{a}} t \\
\sin \omega_{\mathrm{a}} t
\end{array}\right\}
\end{aligned}
$$

où $a_{i j}$ et $b_{i j}$ sont respectivement les coefficients de raideur et d'amortissement du palier relatifs à la position d'équilibre statique du rotor $((i, j)=(X, Y))$.

En variables sans dimension, l'équation matricielle (B1) s'écrit :

$$
\begin{aligned}
{\left[\begin{array}{cc}
\tilde{m} & 0 \\
0 & \tilde{m}
\end{array}\right]\left\{\begin{array}{l}
\tilde{X}^{\prime \prime} \\
\tilde{Y}^{\prime \prime}
\end{array}\right\}+\left\{\begin{array}{ll}
B_{X X} & B_{X Y} \\
B_{Y X} & B_{Y Y}
\end{array}\right\}\left\{\begin{array}{l}
\tilde{X}^{\prime} \\
\tilde{Y}^{\prime}
\end{array}\right\} } \\
+\left[\begin{array}{ll}
A_{X X} & A_{X Y} \\
A_{Y X} & A_{Y Y}
\end{array}\right]\left\{\begin{array}{l}
\tilde{X} \\
\tilde{Y}
\end{array}\right\}=\tilde{m} \varepsilon_{\mathrm{b}}\left\{\begin{array}{l}
\cos \tilde{t} \\
\sin \tilde{t}
\end{array}\right\}
\end{aligned}
$$

avec $\tilde{m}=\frac{m \omega_{\mathrm{a}}^{2} C}{W_{0}} ; A_{i j}=a_{i j} \frac{C}{W_{0}} ; B_{i j}=b_{i j} \frac{C \omega_{\mathrm{a}}}{W_{0}} ; \tilde{t}=\omega_{\mathrm{a}} t$; $\tilde{X}=\frac{X}{C} ; \tilde{Y}=\frac{Y}{C} ; \tilde{X}^{\prime}=\frac{\dot{X}}{C \omega_{\mathrm{a}}} ; \tilde{Y}^{\prime}=\frac{\dot{Y}}{C \omega_{\mathrm{a}}} ;\left(\tilde{X^{\prime \prime}}, \tilde{Y}^{\prime \prime}\right)=$ $\frac{(\ddot{X}, \ddot{Y})}{C \omega_{\mathrm{a}}^{2}} ; \varepsilon_{\mathrm{b}}=\frac{e_{\mathrm{b}}}{C}$. 
Le vecteur solution du système d'équations (B2) est de la forme :

$$
\left\{\begin{array}{c}
\tilde{X} \\
\tilde{Y}
\end{array}\right\}=\left\{\begin{array}{c}
\tilde{X}_{1} \\
\tilde{Y}_{1}
\end{array}\right\} \cos \tilde{t}+\left\{\begin{array}{c}
\tilde{X}_{2} \\
\tilde{Y}_{2}
\end{array}\right\} \sin \tilde{t}
$$

En remplaçant (B3) dans le système (B2) et en identifiant les termes en $\cos \tilde{t}$ et $\sin \tilde{t}$, on aura :

$$
\begin{aligned}
& {\left[\begin{array}{llll}
\left(A_{X X}-\tilde{m}\right) & B_{X X} & A_{X Y} & B_{X Y} \\
-B_{X X} & \left(A_{X X}-\tilde{m}\right)-B_{X Y} & A_{X Y} \\
A_{Y X} & B_{Y X} & \left(A_{Y Y}-\tilde{m}\right) & B_{Y Y} \\
-B_{Y X} & A_{Y X} & -B_{Y Y} & \left(A_{Y Y}-\tilde{m}\right)
\end{array}\right]\left\{\begin{array}{c}
\tilde{X}_{1} \\
\tilde{X}_{2} \\
\tilde{Y}_{1} \\
\tilde{Y}_{2}
\end{array}\right\}=} \\
& \tilde{m} \varepsilon_{\mathrm{b}}\left\{\begin{array}{l}
1 \\
0 \\
0 \\
1
\end{array}\right\}
\end{aligned}
$$

La solution du système (B4) s'écrit :

$$
\left\{\begin{array}{l}
\tilde{X}_{1}=\tilde{m} \varepsilon_{\mathrm{b}} \frac{A C+B D}{C^{2}+D^{2}} \\
\tilde{X}_{2}=-\tilde{m} \varepsilon_{\mathrm{b}} \frac{B C-A D}{C^{2}+D^{2}} \\
\tilde{Y}_{1}=\tilde{m} \varepsilon_{\mathrm{b}} \frac{F C+G D}{C^{2}+D^{2}} \\
\tilde{Y}_{2}=-\tilde{m} \varepsilon_{\mathrm{b}} \frac{G C-F D}{C^{2}+D^{2}}
\end{array}\right.
$$

avec

$$
\begin{aligned}
l A= & -\tilde{m}+A_{Y Y}-B_{X Y} \\
B= & A_{X Y}+B_{Y Y} \\
C= & \tilde{m}^{2}-\tilde{m}\left(A_{X X}+A_{Y Y}\right)+A_{X X} A_{Y Y}-A_{X Y} A_{Y X} \\
& -\left(B_{X X} B_{Y Y}-B_{X Y} B_{Y X}\right) \\
D= & -\tilde{m}\left(B_{X X}+B_{Y Y}\right)+A_{X X} B_{Y Y}+A_{Y Y} B_{X X} \\
& -A_{X Y} B_{Y X}-A_{Y X} B_{X Y} \\
F= & -A_{Y X}+B_{X X} \\
G= & \tilde{m}-A_{X X}-B_{Y X}
\end{aligned}
$$

La solution (B3) du système (B2) correspond à une trajectoire de forme elliptique (Fig. B1) dont les caractéristiques sont les suivantes :

$a=\tilde{a} C:$ demi-grand axe;

$b=\tilde{b} C:$ demi-petit axe;

$\varphi$ : angle de déphasage entre le vecteur balourd et le vecteur déplacement;

$\delta^{*}$ : l'angle qui donne l'inclinaison du demi-grand axe par rapport à l'axe $X$.

Ainsi, dans le repère propre de l'ellipse $\left(o_{a 0}, X *, Y *\right)$, l'équation de l'ellipse s'écrit :

$$
\left\{\begin{array}{l}
\tilde{X} *=\tilde{a} \cos (\tilde{t}+\varphi) \\
\tilde{Y} *=\tilde{b} \sin (\tilde{t}+\varphi)
\end{array}\right.
$$

où : $(\tilde{X} *, \tilde{Y} *)=\frac{(X *, Y *)}{C}$

Compte tenu de la relation (B6), la projection sur

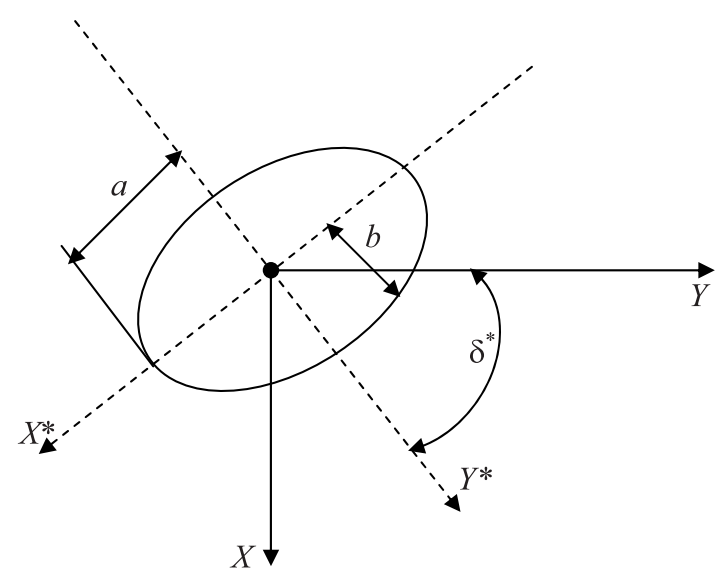

Fig. B1. Caractéristiques géométriques de la trajectoire elliptique du centre de l'arbre.

$\left(o_{a 0}, X, Y\right)$ donne :

$$
\left\{\begin{array}{l}
\tilde{X}=\tilde{a} \cos (\tilde{t}+\varphi) \cos \delta^{*}+\tilde{b} \sin (\tilde{t}+\varphi) \sin \delta^{*} \\
\tilde{Y}=-\tilde{a} \cos (\tilde{t}+\varphi) \sin \delta^{*}+\tilde{b} \sin (\tilde{t}+\varphi) \cos \delta^{*}
\end{array}\right.
$$

Les équations (B3) sont identiques aux équations (B7), ce qui permet d'écrire :

$$
\left\{\begin{array}{c}
\tilde{X}_{1} \cos \tilde{t}+\tilde{X}_{2} \sin \tilde{t}=\tilde{a} \cos (\tilde{t}+\varphi) \cos \delta^{*} \\
+\tilde{b} \sin (\tilde{t}+\varphi) \sin \delta^{*} \\
\tilde{Y}_{1} \cos \tilde{t}+\tilde{Y}_{2} \sin \tilde{t}=-\tilde{a} \cos (\tilde{t}+\varphi) \sin \delta^{*} \\
+\tilde{b} \sin (\tilde{t}+\varphi) \cos \delta^{*}
\end{array}\right.
$$

Ce système d'équations étant vérifié quelque soit le temps $\tilde{t}$, il vient en identifiant les termes en $\cos \tilde{t}$ et $\sin \tilde{t}$ :

$$
\left\{\begin{array}{l}
\tilde{X}_{1}=\tilde{a} \cos \delta^{*} \cos \varphi+\tilde{b} \sin \delta^{*} \sin \varphi \\
\tilde{X}_{2}=-\tilde{a} \cos \delta^{*} \sin \varphi+\tilde{b} \sin \delta^{*} \cos \varphi \\
\tilde{Y}_{1}=-\tilde{a} \sin \delta^{*} \cos \varphi+\tilde{b} \cos \delta^{*} \sin \varphi \\
\tilde{Y}_{2}=\tilde{a} \sin \delta^{*} \sin \varphi+\tilde{b} \cos \delta^{*} \cos \varphi
\end{array}\right.
$$

La résolution des équations (B9) permet de déterminer les caractéristiques de l'ellipse :

$$
\left\{\begin{aligned}
\tilde{a} & =\left(\frac{\left(\tilde{X}_{1}^{2}+\tilde{X}_{2}^{2}+\tilde{Y}_{1}^{2}+\tilde{Y}_{2}^{2}\right)}{2}\right. \\
& \left.+\sqrt{\left(\tilde{X}_{1} \tilde{Y}_{1}+\tilde{X}_{2} \tilde{Y}_{2}\right)^{2}+\frac{1}{4}\left(\tilde{X}_{1}^{2}+\tilde{X}_{2}^{2}-\tilde{Y}_{1}^{2}-\tilde{Y}_{2}^{2}\right)^{2}}\right)^{\frac{1}{2}} \\
\tilde{b} & =\left(\frac{\left(\tilde{X}_{1}^{2}+\tilde{X}_{2}^{2}+\tilde{Y}_{1}^{2}+\tilde{Y}_{2}^{2}\right)}{2}\right. \\
& \left.-\sqrt{\left(\tilde{X}_{1} \tilde{Y}_{1}+\tilde{X}_{2} \tilde{Y}_{2}\right)^{2}+\frac{1}{4}\left(\tilde{X}_{1}^{2}+\tilde{X}_{2}^{2}-\tilde{Y}_{1}^{2}-\tilde{Y}_{2}^{2}\right)^{2}}\right)^{\frac{1}{2}} \\
\delta^{*} & =\frac{1}{2} \tan ^{-1}\left(\frac{-2\left(\tilde{X}_{1} \tilde{Y}_{1}+\tilde{X}_{2} \tilde{Y}_{2}\right)}{\tilde{X}_{1}^{2}+\tilde{X}_{2}^{2}-\tilde{Y}_{1}^{2}-\tilde{Y}_{2}^{2}}\right) \\
\varphi & =\frac{1}{2} \tan ^{-1}\left(\frac{-2\left(\tilde{X}_{1} \tilde{X}_{2}+\tilde{Y}_{1} \tilde{Y}_{2}\right)}{\tilde{X}_{1}^{2}-\tilde{X}_{2}^{2}+\tilde{Y}_{1}^{2}-\tilde{Y}_{2}^{2}}\right)
\end{aligned}\right.
$$




\section{Références}

[1] V.K. Stokes, Couple stresses in fluids, Physics of fluids 9 (1966) 1709-1715

[2] T.T. Ariman, N.D. Sylvester, Microcontinuum fluid mechanics, A Review, Int. J. Eng. Sci. 11 (1973) 905-930

[3] T.T. Ariman, N.D. Sylvester, Application of microcontinuum fluid mechanics, J. Eng. Sci. 12 (1974) 273-293

[4] J.R. Lin, Squeeze film characteristics of long partial journal bearings lubricated with couple stress fluids, Tribology Int. 30 (1997) 53-58

[5] J.R. Lin, C.B. Yang, R.F. Lu, Effects of couple stresses in the cyclic squeeze films of finite partial journal bearings, Tribology Int. 34 (2001) 119-125

[6] J.R. Lin, Squeeze film characteristics of finite journal bearings : couple stress fluid model, Tribology Int. 4 (1998) 201-207

[7] D.R. Oliver, Load enhancement effects due to polymer thickening in a short model journal bearings, J. Non Newtonian Fluid Mech. 30 (1988) 185-196

[8] U.M. Mokhiamar, W.A. Crosby, H.A. El-Gamal, A study of a journal bearing lubricated by fluids with couple stress considering the elasticity of the liner, Wear 224 (1999) 194-201

[9] M. Lahmar, Elastohydrodynamic analysis of doublelayered journal bearings lubricated with couple-stress fluids. Proc. I Mech E, Part J: J. Eng. Tribology 219 (2005) 145-171

[10] J.R. Lin, Linear stability analysis of a rotor bearing system : couple stress fluid model, Computers \& Structures 79 (2001) 801-809

[11] X.L. Wang, K.Q. Zhu, S.Z. Wen, Thermohydrodynamic analysis of journal bearings lubricated with couple-stress fluids, Tribology Int. 34 (2001) 335-343

[12] X.L. Wang, K.Q. Zhu, S.Z. Wen, On the performance of dynamically loaded journal bearings lubricated with couple-stress fluids, Tribology Int. 35 (2002) 185-191

[13] D. Nicolas, Paliers hydrodynamiques soumis à un torseur de forces quelconques, Thèse de doctorat, INSA, Lyon, 1972

[14] D.G. Christopherson, A new mathematical method for the solution of oil film lubrication problems, Proc. Int. Mech. Eng. 146 (1941) 126-135 